\title{
Prediction and detection in change of cognitive load for VIP's by a machine learning approch
}

\author{
by \\ Fahim Rahman \\ 17101500 \\ Md.Istiyak Ahmed \\ 16201021 \\ Saif Shahnewaz Saad \\ 16101181 \\ Md Ashrafuzzaman \\ 16101110 \\ Sharita Shehnaz Mogno \\ 21141040
}

A thesis submitted to the Department of Computer Science and Engineering in partial fulfillment of the requirements for the degree of B.Sc. in Computer Science

Department of Computer Science and Engineering

Brac University

June 2021

(C) 2021. Brac University

All rights reserved. 


\section{Declaration}

It is hereby declared that

1. The paper submitted is our own original research completed while pursuing a degree at Brac University.

2. Except as properly referenced by complete and correct referencing, the study does not include content previously published or written by a third party.

3. The paper does not contain any content that has been approved or applied for any particular academic or other institution's degree or certificate.

4. We have acknowledged all main sources of help.

\section{Student's Full Name \& Signature:}

Fahim Rahman

17101500

Saif Shahnewaz Saad

16101181
Md.Istiyak Ahmed 16201021

Md Ashrafuzzaman

16101110

Sharita Shehnaz Mogno

21141040 


\section{Approval}

The thesis/project titled "Prediction and detection in change of cognitive load for VIP's by a machine learning approch" submitted by

1. Fahim Rahman (17101500)

2. Md. Istiyak Ahmed (16201021)

3. Saif Shahnewaz Saad (16101181)

4. Md Ashrafuzzaman (16101110)

5. Sharita Shehnaz Mogno (21141040)

Of Spring, 2021 has been accepted as satisfactory in partial fulfillment of the requirement for the degree of B.Sc. in Computer Science on June 2, 2021.

\section{Examining Committee:}

Supervisor:

(Member)

Dr. Mohammad Zavid Parvez

Associate Professor

Department of CSE

BRAC University

Co-Supervisor:

(Member)

Rafeed Rahman

Lecturer

Department of CSE

Brac University

Head of Department:

(Chair)

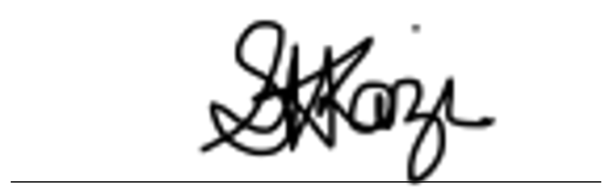

Sadia Hamid Kazi

Chairperson

Department of Computer Science and Engineering

BRAC University 


\section{Ethics Statement}

Our research is of the highest quality and integrity. The privacy and integrity of our research paper participants are valuable. We have done our research in an unbiased manner so our analysis is independent and impartial. Hopefully, in the future, this research analysis will add some extra feature to humankind for their development 


\section{Abstract}

The significance and urgency of detecting cognitive load of Visually Impaired Person is essential when perception comes while designing an automated navigation aid for them in unfamiliar indoor environments.Our paper presents a novel, robust and multidimensional framework based on iterative feature pooling technique which recursively selects paramount features that maintains relation with the change in cognitive load of the brain. We have chosen to use Electroencephalogram as it is one of the fastest imaging techniques available having a high sampling rate and analytical neuro-psychologic benchmarks of perceptive process indicated by rhythmic activities of the brain. We took the well established ERDS method for indexing the cognitive load and further developed the work by operating with the band power of not only the Alpha wave but the Alpha Beta ratio band power and Alpha Theta ratio band power. The intricacy of the tasks in terms of cognitive load were quantified considering multiple aspects to support the redemption of usability of a way finding aid by features extraction from specific attributes, some of which were new to this field, to support the vindication of accessibility of a way finding aid.As the machine learning classifier the Gradient Boost outperformed all other classifiers(94\% accuracy). We considered other performance parameters like the $\mathrm{f}-1$ score,recall, time delay, sensitivity and false positive rate to evaluate the performance of all available supervised ML classifiers. This chapter marks out the estimation of based on existing literature, background, leeway, characteristics, and machine learning approaches, cognitive load was calculated using EEG data.

Keywords: Cognitive load; Machine Learning; Supervised Learning; EEG; Performance parameters; Alpha Beta ratio ; Gradient Boost Algorithm; ERDS 


\section{Acknowledgement}

First and foremost, all praise to Almighty Allah for keeping us safe and sound in between this Covid pandemic so that we could begin the study work and bring in our best efforts to successfully complete it without any major difficulties. Second, we'd like to express our gratitude and admiration for our honorable supervisor, Dr. Mohammad Zavid Parvez, for his tremendous efforts, unrivalled encouragement, and unwavering support during the research. He was still willing to assist us anytime we wanted it. We are incredibly fortunate and humbled to have him as our supervisor. Moreover, without our parents' unwavering support, it may not be possible. We are now on the brink of graduating thanks to their generous encouragement and prayers. Last but not least, we would like to express our gratitude to our very own BRAC University for allowing us to perform this thesis. 


\section{Table of Contents}

Declaration $\quad$ i

Approval ii

Ethics Statement iii

Abstract

$\begin{array}{ll}\text { Dedication } & \text { v }\end{array}$

Acknowledgment $\quad$ v

Table of Contents vi

List of Figures viii

List of Tables ix

Nomenclature $\quad$ xi

1 Introduction $\quad 1$

1.1 Motivation . . . . . . . . . . . . . . . . 3

1.2 Aims and Objectives . . . . . . . . . . . . . . . . . 3

1.3 Thesis Orientation . . . . . . . . . . . . . . . . 4

2 Literature review $\quad 5$

3 Background studies 14

3.1 EEG Signals . . . . . . . . . . . . . . . . . . . . . . . . . 14

3.2 CLI . . . . . . . . . . . . . . . . . . . . . . . . 15

3.3 Brain Waves . . . . . . . . . . . . . . . . . . 15

3.3 .1 Gamma waves . . . . . . . . . . . . . . . . 16

3.3 .2 Beta waves . . . . . . . . . . . . . . . . 16

3.3.3 Alpha waves . . . . . . . . . . . . . . . . . . . 16

3.3 .4 Theta waves . . . . . . . . . . . . . . . . 17

3.3.5 Delta waves . . . . . . . . . . . . . . . . 17

3.4 Wavelet Transform . . . . . . . . . . . . . . . . 17

$3.4 .1 \mathrm{DWT}(\mathrm{db} 4) \ldots \ldots \ldots \ldots 1 . \ldots \ldots$

3.5 Fourier Transform . . . . . . . . . . . . . . . . . . . . . . . . . . . . . . . . . 18

$3.5 .1 \quad \mathrm{FFT} \ldots \ldots \ldots \ldots \ldots \ldots$ 


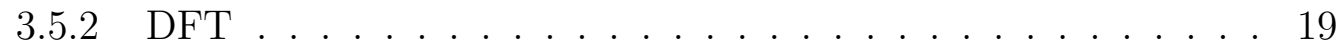

3.5.3 IFFT . . . . . . . . . . . . . . . . . . 19

3.6 Machine Learning: . . . . . . . . . . . . . . . . . . . . . . 20

3.6.1 Decision Tree . . . . . . . . . . . . . . . . . 20

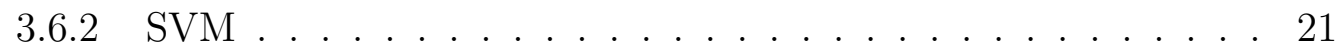

3.6.3 Random Forest . . . . . . . . . . . . . . . . . . . . . . . . . 22

3.6.4 Gradient Boost . . . . . . . . . . . . . . . . . 22

3.6.5 Naive Bayes . . . . . . . . . . . . . . . . . . 23

3.6 .6 K-Nearest Neighbour . . . . . . . . . . . . . . . . . . 24

3.6.7 Logistic Regression . . . . . . . . . . . . . . . . . . 25

3.6.8 Stocastic gradient Descent . . . . . . . . . . . . . 26

$3.6 .9 \mathrm{MLP} \ldots \ldots \ldots \ldots \ldots . \ldots \ldots 27$

4 Experimental setup $\quad 28$

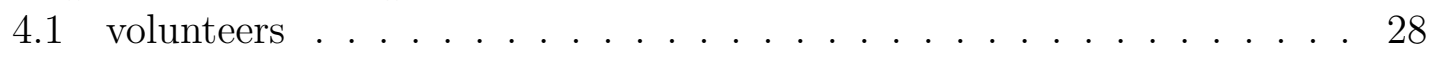

4.2 The Indoor routes . . . . . . . . . . . . . . . . . . . . . . . . . . . . . . . . . . . . . . . . . . . . . . . . . .

4.3 Data Acquisition . . . . . . . . . . . . . . . . . . . . . . . . 29

5 Methodology $\quad 31$

5.1 System Work Flow . . . . . . . . . . . . . . . . . . 31

5.2 Pre-Processing of the signals . . . . . . . . . . . . . . . . . . 32

5.3 Band Extraction . . . . . . . . . . . . . . . . . . . . . 33

5.4 Feature Extraction . . . . . . . . . . . . . . . . . . 33

5.4.1 The ERD/ERS Calculation by a novel approch: . . . . . . . . 37

5.4 .2 Creation of the dynamic feature pool: . . . . . . . . . 37

5.4 .3 Classification: . . . . . . . . . . . . . . . . . 38

6 Results and Discussion $\quad 40$

6.1 A Comparative study: . . . . . . . . . . . . . . . . 50

6.2 Future Works: . . . . . . . . . . . . . . . . . . . . 50

6.3 Conclusion: . . . . . . . . . . . . . . . . . 51

$\begin{array}{ll}\text { Bibliography } & 58\end{array}$ 


\section{List of Figures}

3.1 EEG Processing . . . . . . . . . . . . . . . . . . . . . . . 14

3.2 Decision tree example . . . . . . . . . . . . . . . . . . . . . 21

3.3 K-nearest neighbor . . . . . . . . . . . . . . . . . . . . . 24

3.4 Multi layer Perception Layer . . . . . . . . . . . . . . . . . 27

4.1 The Emotiv Epoc+ and the placement of electrodes . . . . . . . . . 30

5.1 System work Flow $\ldots \ldots \ldots \ldots$

6.1 confusion matrix at door . . . . . . . . . . . . . . 42

6.2 confusion matrix at elevator $\ldots \ldots \ldots \ldots \ldots$

6.3 confusion matrix at narrow . . . . . . . . . . . . . . 43

6.4 confusion matrix at open . . . . . . . . . . . . . . 43

6.5 confusion matrix at stairs $\ldots \ldots \ldots \ldots \ldots \ldots 4$

6.6 Main Feature at $\mathrm{AB} \ldots \ldots \ldots \ldots$

6.7 Main Feature at AT . . . . . . . . . . . . . 45

6.8 classifier selection from alpha-beta bands ratio . . . . . . . . . 45

6.9 classifier selection from alpha-theta bands ratio $\ldots \ldots \ldots . . . .46$

6.10 features selection in alpha-beta band ratio . . . . . . . . . 46

6.11 features selection in alpha-theta band ratio . . . . . . . . . 47

6.12 ROC curve for the Alpha-Beta ratio . . . . . . . . . . . . . . 47

6.13 ROC curve for the Alpha-Beta ratio . . . . . . . . . . . . . 48

6.14 ROC curve for the Alpha-Beta ratio . . . . . . . . . . . . . . . . 48

6.15 ROC curve for the Alpha-Theta ratio . . . . . . . . . . . . . 49 


\section{List of Tables}

4.1 Category of volunteers . . . . . . . . . . . . . . . . . . 28

4.2 Indoor Mobility challenges . . . . . . . . . . . . . . . . . . . . . 29

6.1 Performance test(Accuracy,Sensitivity,Specificity) of the GB classifier in different obstacles . . . . . . . . . . . . . . . . . . . . . 41

6.2 Performance test(Null Accuracy,False Alarm,Time Delay,Quality Value) of the GB classifier in different obstacles . . . . . . . . . . . . . . 41

6.3 Changes in the performance of the GB as we iterate over the number of estimators . . . . . . . . . . . . . . . . . 42 


\section{Nomenclature}

The next list describes several symbols \& abbreviation that will be later used within the body of the document

$A M D$ Advanced Micro Devices

$A U C$ Area Under the Curve

$B V P$ Blood volume pulse

CLT Cognitive load theory

DFT Discrete Fourier Transform

DWT Discrete Wavelet Transform

EEG Electroencephalography

ERDS Event Related De Synchronization Synchronization

FFT Fast Fourier Transformation

$F N \quad$ False Negative

FP False Positive

GB Gradient Boosting

IFFT Inverse Fast Fourier transform

$K N N$ K Nearest Neighbors

$L R \quad$ Logistic Regression

MLP Multilayer Perceptron

$N B \quad$ Naive Bayes

RF Random Forest

$R O C$ receiver operating characteristic

$S F D R$ Spurious free dynamic range

$S G D$ Stochastic Gradient Descent

SVM Support Vector Machines 
$T N \quad$ True Negative

TP True Positive

VIP Visually Impaired Person 


\section{Chapter 1}

\section{Introduction}

Around 285 Million in today's world suffer from loss of sight according to World Health Organisation. These people day to day go through a tremendous amount of sufferings for their disability. It is quite easy to walk ourselves through the picture of their indoor mobility struggle. On top of that they are not few in number. Rogers et $\mathrm{Al}$. have stated that there is nearly $63 \%$ probability of dementia for sight loss people over eight and half years. It can happen for various reasons. Initially Optic Neuritis, Congenital Glaucoma and Cataracts can cause temporary or parmanent blindness. Science has come up with many visual and navigation aids which assist disabled people to lead a safer and easier daily lives. But this study of ours is based on the EEG signal from the brain analysing which navigation of visually impaired persons in surrounding areas is helped. Cognitive load is measured to let them know a smoother path for easier navigation. There are many navigation aids which take into count the cognitive load in the process such as ours [12]. In our study we have magnified the accuracy of the result by determining which feature is best in different situations where the main purpose is assisting the visually impaired people by developing a helpful system to help in navigation and obstacle detection.

Previously in the past studies regarding the visually impaired people we have seen them to use eeg signals like behavioral, electroencephalography, Peripheral Biosignals, multimodal biosignals to determine cognitive load and assess in navigation. And as for classifier they have frequently used random forest classifier. In our study we have seen its effectiveness too. It takes into consideration the Correlated predictor variables and able to reach a simple up to the mark solution using cognitive load. Fast Fourier Transform is used heavily for the data sample manipulation which is present in our study too [83].

The theory that provides ways to determine instructional model and cognitive process is Cognitive load theory. (CLT) enables the process measuring the cognitive architecture and the information structure . Cognitive load theory enhances effectiveness as well as accuracy. It diminishes the limitation of people's cognitive processing ability. Cognitive architecture that used to give effective instructional strategies is the basis of Cognitive load theory [56]. Limited working memory is handled by cognitive architecture, which includes somewhat autonomous visual and auditory processing units that's linked to an infinite long-term memory. On the contrary, the work expended in working memory for the total mental activity to 
complete is the cognitive load. In other way, the resources that have been utilised from working memory is cognitive load. The three categories are intrinsic, germane and extraneous cognitive load. The effort or work put into a particular topic indicates Intrinsic cognitive load. Extraneous means the process through which tasks are represented for a learner and the work that puts in for creation of a stable repository of information is referred to as germane cognitive load [26]. John Sweller conducted a study of problem solving in the late 1980s, which led to the development of cognitive load theory. Sweller claims that design of teaching can assist learners mitigate cognitive load. Afterwards, other researchers devised a method for determining mental effort as observed, which is a good indicator of cognitive load. The fast reaction caused by task is a sensitive and reliable indicator of cognitive stress that is linked to working memory. Working memory, on the other hand, is severely constrained in terms of capacity and length. Under some circumstances, these limits will obstruct learning. Task completion might be hampered by a high cognitive load. Therefore, It's crucial to remember that everyone's experience with cognitive load is different [21].

Cognitive load is a conceptual thoery that is becoming more and more important in educational study. Working memory capacity is finite which is the primary premise of cognitive load theory, hence learning would be impaired if a learning activity takes too much capacity [71]. Creating learning process that maximize the utilization of ability of working memory while avoiding cognitive overabundance is the proposed cure. Cognitive load theory has made significant contributions to educational research and has been used to explain a broad range of experimental results.LSTM network is a recurrent neural network that may learn order dependency in sequence prediction challenges. This is needed in a variety of complicated issue areas, including machine translation, speech recognition, and others.Deep learning's LSTMs are a complicated topic. It might be difficult to grasp the concept of LSTMs and how words like bidirectional and sequence-to-sequence apply to the area [41].

ERD and ERS can occur as a result of sensory, cognitive, and motor processing. ERD is event related desynchronization. On the other hand, ERS means event related synchronization. Both phenomena are frequency-band specific and are locked by time but not phase to the occurrence [14]. ERD/ERS spatial mapping can be utilized to investigate the characteristics of cortical activation design. Sensory and cognitive processing as well as motor behavior cause ERP which means event related potential and slight variations in ERD and ERS. The previous is a rhythmic activity amplitude drop that is short-lived and limited, whereas the later is an amplitude rise. ERPs, on the other hand, are phase-locked responses of bio electrical brain activity that may be described by adding synaptic potentials [11].

Previously cognitive load was measured by many different ways, Subjective measurement of cognitive load was one of the most recurrent procedure, where each participants in the experimental setup would submit a self-reported rating scale for the appraisal of the mental load frequently combined with a estimation of subjectively perceived task difficulty [7]. The scale method is based on the postulations that probationers can assemble a well founded and valid estimation of the consignment of load they were defy ed with in a particular situation. The rating scales illustrates the utility of this routine for CLT research for many experiments[38]. For cognitive 
load many objective index have been developed in this field they act as the barometers other than the subjective measurement approaches. They can be discerned by their connection to the learning process in follow-up variables (learning outcomes), stored variables (task hassles), and activity-related bohemianism intimation (e.g., immaterial criterion or time on task) [53] [38]. As for our studies we used the alpha beta band power ratio for the ERDS to index the cognitive load and did a binary classification with the ensemble machine learning classifier, the feature pool that we created proved to be very very efficient for pointing out the best features that has a co-relation with the cognitive load of the VIP's who volunteered in our research.As a result a satisfying accuracy performance index was achieved.

\subsection{Motivation}

Dealing with vision loss or low vision is just one of the obstacles that the visually impaired face on a daily basis. Visually impaired people are similar to Normal people but they just they have trouble having a clear vision of the surroundings. A quick glance about us reveals how visual the majority of the information in our surroundings is.Near or far vision impairment affects at least 2.2 billion individuals worldwide (source:WHO). These people consider themselves as useless or live in such a state of life where they try to isolate themselves from the rest of the normal world.Keeping all these in our mind this particular topic has been a thing of interest to us. Through our study if we can perfectly design a navigating system for the VIP's in indoor mobility so that their life will become a bit easier because they will not have to depend on a third person to move around indoor environment. We also wanted to contribute in the existing literature and work done by other researchers by pointing out significant features,methodologies so that future works on these fields will have a boost up through our work. The core inspiration that triggered our work was the empathy we felt towards the VIP(Visually Impaired Person) the automated navigation aid designed on the basis of detecting their cognitive load would help them to achieve an overall conception around their indoor environment as well as guide through different obstacles avoiding any kind of major or minor accidents.

\subsection{Aims and Objectives}

These are the driving factors that triggered our current work with existing resources.

- Exploring the EEG of Visually Impaired Persons to detect the cognitive load under certain conditions in indoor environment.

- Present a novel "feature-pooling" based framework that appraise the change in brain signal pattern of the VIPs which can be used to design real-time based navigation aid in indoor mobility

- Point out the environmental factors that trigger cognitive load

- Detecting the most significant features by recursive technique that fluctuates with the change of brain activity that increases cognitive load

- Comparing all Supervised Machine learning classifiers to find the best one for the prediction of serving our purpose. 


\subsection{Thesis Orientation}

Our whole thesis/work is described in six chapter, and they are:

Chapter 1 talks about the introduction to the Visually Impaired people, different kinds of cognitive load, detection through EEG signals and the connection between them all. Our aims and objectives along with the motivation is clearly stated here.

In Chapter 2, there is a detailed description about the existing work and literature review regarding various methodologies and features that are closely associated with Cognitive load, VIPs and EEG signals. What is the measuring index of cognitive load is also described in this chapter briefly

In chapter 3 we tried to throw light on some of the topics that were used in our thesis and how that particular part is connected to our thesis.A major breakdown of all the supervised learning classifiers and their usability if given in this chapter.

In chapter 4, a descriptive writing about the data-set, participants and experimental setup can be found.We manually did not collect the data so detailed information if given how each data part works.

In chapter 5, there is the system work flow and detailed methodology, how all the works have been done along with information of each feature that we extracted.Then how the data-set is prepared for the classifier and where lies our novelty of the work is also mentioned in the later part of the chapter.

In chapter 6 , the result we got from the classifier and out personal observation of different classifiers and situation is compared in different tables and visualization. The chapter holds a comparative study with the existing method against our proposed method that indicates the superiority of our study, finally we concluded by stating our flaws and future work. 


\section{Chapter 2}

\section{Literature review}

There has been done a lot of thesis work and research based on EEG signals from time to time. A parallel method framework for extracting features of EEG data on MPI is described in the study. The major goal was to integrate an MPI (Message Passing Interface) based parallel framework to the classical welch approach for bigger datasets in order to obtain power spectrum properties of EEG signals and speed up brain signal processing. The PSD (power spectral density) of those $\mathrm{N}$ segments is computed in parallel by certain nodes, the findings are gathered and processed by the master node, and (this is the uniqueness) may be stored as a text file. EEG of every channel is separated into $\mathrm{N}$ overlapping data segments.Despite the fact that many methods of extracting features were mentioned, the final PSD of each channel collected in text file could be studied and analyzed by MS Excel, which is 7 times faster than Python default library and allows even inexperienced users (in the sector of parallel processing) to use this framework.A low-end pc can handle the framework and a window function (Hamming window) was used to prevent spectral leakage, then FFT was used in the welch method, one key point is cooperating with each node, evenly distributed tasks to each node because this directly affects the performance of the algorithm as they tested accuracy, speed and speedup for performance test. They described PSD as the best for EEG feature extraction method and when fused with MPI, according to them gives the best result[86].

Another article employed an EEG feature derived from a bilevel network, a minimal spanning tree, and a regional network, and the average accuracy rate was $89.50 \%$. Because current approaches for brain network analysis are restricted by size of the network, complexity, and standardization, this research proposes a unique composite feature based on bilevel network analysis to extract brain function. To tackle the global network connection issue, the first network layer uses a minimal spanning tree depending on EEG signal nodes in distinct MIs, while the second layer uses a regional network in distinct movement patterns to define network features. The MST basic properties are combined with the regional network's directional properties to create a vector.For feature extraction, the Pearson correlation coefficient is chosen, and MST features are chosen using the Kruskal method.The first layer's characteristics are diameter and average eccentricity; additional characteristics are average node degree, average clustering, and average path length.Because MST lacks the small world network characteristic, the regional network developed in this research aids in the more thorough characterization of EEG data. 
Regional networking can manage data clustering better than MST alone, thus the two are combined, which is the paper's originality.They may be fused using serial combination and a Bayesian Fusion technique based on weighted average, which decreases subject miscalculation. The other methods mentioned here are coherence spectrum, mutual information. The classification technique included Sparse Bayesian Learning (SBL), Filter Bank common spatial pattern (FBL), Channel Optimization based on Lp-norm (COL)[81].

The cognitive changes in wakefulness and sleepiness state of motorists can be detected by EEG signals using Tunable Q factor wavelet transform (TQWT) in a study by decaying the signal into sub - band, which were then used for extracting features of the Hjorth movement such as minimum value, upper value, mean and SD, and classification methods such as logistic fine gaussian SVM, weighted KNN, decision trees, ELM, logistic regression. The Kruskal-Wallis test and ELM with an accuracy of $91.842 \%$ are used to measure alertness and sleepiness state EEG signals, as well as constructed enhanced trees. This paper also reviewed some methods for EEG based alertness and drowsiness states like FFT based features, nine-dimensional feature vectors with 2 classifiers SVM and ANN (acc-83.5\%, outperformed others).

In other papers, Respiratory Rate Variability signal analysis, alpha rhythm of EEG signal, spectral features for same purpose and WT to divide the bands.Empirical mode reduction employs AM-FM components to extract rhythm-based information, which are then used with ANN to categorize Higuchi's Petrosian's fractal dimension, In another research, Parseval's energy theorem was used to create an energy co-efficient that was amended by input-output clustering and used to an ANN classifier.PSD with WT, RR time series was decomposed using iterative filtering procedure into intrinsic mode function utilizing Adaptive Hermite Decomposition (AHD) based features with ELM classifier, alpha and theta rhythms, In other works, determinism characteristics from recurrence qualification analysis with SVM and Bayer's classifier were employed, with the same goal of detecting sleepiness. They utilized the KW (for testing features like Hjorth mobility, mean, maxima, minima, and SD) because it is a non-parametric approach that does not require the normal distribution assumption for test samples for feature testing. This calculates probiotic values of Kruskal Wallis test in each sub bands. This was their novelty which resulted in better accuracy than any of their previous works[74].

Linear Discriminant Analysis (LDA) was utilized in another article for feature selection and dimensionality reduction, with the informative and discriminative twodimension features serving as a baseline for classification using a Multi-Layers Perceptron (MLP) neural network. The proposed model achieves a high sensitivity, specificity and accuracy of $100 \%$. Principal component analysis is used for this (PCA), independent component analysis (ICA), LDA and K-means clustering were applied and the most prominent and discriminative features were used as inputs of a kernel machine for the classification decision at the last step. Two mostly used kernel machines with different architectures were proposed, such as artificial neural network (ANN)[18][33] and support vector machine (SVM)[25]. Most of these studies focus on epilepsy by using a feature vector with at least three dimensions 
as inputs of a complex kernel machine. WT is also used in this study to investigate EEG signals in the time-frequency domain. EEG data is projected into a collection of wavelet coefficients that show the correlation between the signals and their associated EEG cycles. Furthermore, Areas under the frequency spectrum of each wavelet coefficient are used as the features. These areas are calculated using trapezoidal rule from numerical analysis. For feature extraction, each wavelet coefficient was transformed into its time domain and applied Fourier transform to the wavelet coefficient to obtain the frequency spectrum. Compared to other works, in general for different experiments; The suggested model developed in this study reaches a high level of accuracy. The result of EEG signal classification through MLPNN has demonstrated that through LDA extraction of features can help classifiers perform better while lowering their computing costs.[73].

A further study built an automatic FO EEG signals categorization approach using the Fourier-Bessel series expansion (FBSE) based flex scheduling span wavelet transform. Properties such as Mixture Correntropy (MC) and Empirical Energy (EE) were utilized to classify FO EEG data. A least-squares support vector machine (LSSVM) classifier with 10-fold cross-validation was used to accomplish the assessment job. The established automated method was also improved using a feature ranking mechanism based on probability $(\mathrm{p})$ values. This created automated technique acquired the maximum classification performance metrics such as accuracy (ACC), sensitivity(SE), and specificity (SP) of 95.85 percent, 95.47 percent, and 96.24 percent, respectively. In this study, an automated classification system that accurately classifies FO EEG signals using high-level signal processing and machine learning strategies was applied, as well as a segment of FO EEG signals chosen at random. For the development of the suggested methodology, EEG signals in NFO pairs were employed.

Following that, the created methodology was tested using 3750 FO and 3750 NFO pairings of EEG signals to identify FO epileptic regions. The proposed methodology incorporates a non-stationary signal processing technique based on the FBSE flexible time-frequency coverage wavelet transform, a features extraction technique, and a machine learning technique to disintegrate the EEG signal into distinct sub-band signals. In our study, we used a learning approach-based To categorize FO and NFO EEG data, an LS-SVM classifier was used. The MC is used to analyze the nonlinear similarity between two random variables with the assistance of two Gaussian kernel functions, and they expanded the work with final selected feature parameters developed using the grid search approach for a broad number of databases. After that, as the number of characteristics grows, it decreases, but the optimum AC, SE, and SP for a matrix of feature space containing 7500 rows and 30 columns are 95.85 percent, 95.47 percent, and 96.24 percent, respectively. In a recent study, With a small section of FO and NFO EEG impulses (length of 2048 samples), the authors used FBSE-EWT and a Sparse Auto Encoder (SAE) based support vector machine (SAE-SVM) classifier to declare a classification accuracy of 100 percent.[78].

Several characteristics and data manipulations have been investigated in a review study to identify, evaluate, and categorize seizures using EEG signals. The main aim of the paper was to make it easy for the new learners to study in this field as 
there is similar work done in many papers and this paper does a comparison study of all methods, features and classifiers to choose the best and this was the novelty of the paper. For feature selection (the significance of each features), they used the Bayesian error and nonparametric probability distribution estimation. Variance, non-linear energy, Shannon entropy from raw EEG, kurtosis and line-length calculated on WT. When these compared in Bayesian method, the result improved (4.77-13.51) \% than normal methods to detect seizure. features with the highest rankings were- Reyne's sample, spectral entropy, non-linear features were Hjorth parameter, entropy, fractal dimension, the focal and the non-focal, time-frequency and time scale features were spectrogram, Extended modified etc.

Compact kernel distribution, Wigner Ville distribution, scalogram, affine WVD, pseudo smoothed AWVD, and CKD with classifier are all examples of distributions. The features were hurst exponent, time-domain feature (elliptical mode decomposition, approximate entropy, weighted-multiscale Reyne, permutation entropy). In some papers GDR, FOR was used to detect seizure.ReliefF, quick correlation basedfilter, and correlation based selection (CFS) are used for feature selection (when used with ANN for the filtered features achieved best result with $91 \%$ sensitivity and $95 \%$ specificity).Based on their experiment utilizing the traditional Bayes classifier, they found that variance, energy, NE, and ShEn obtained from raw EEG signals were effective for classifying between seizure and normal EEG signals, but that they could improve detection performance from the baseline condition. Other TDFs and FDFs, on the other hand, performed poorly in terms of identifying seizures. Furthermore, at some levels, features derived from DWT coefficients provided fundamentally relevant information about seizures that could not be retrieved from raw EEG signals.Moreover, based on redundancy analysis, the energy and variance from the DWT coefficients of distinct decomposition levels were the most important characteristics extracted from CFS.

The energy and variance produced from the DWT coefficients were usually suggested for use in both online and offline AESD because of their relevance and inexpensive processing cost.[75].Some researchers look at spectrum, connectivity, and microstate analysis, as well as the most often used EEG metrics, practical difficulties, and data visualization. Finally, sophisticated approaches such as nonlinear brain dynamics, complex networks, and machine learning are briefly discussed.They explain three major important points of EEG which are- 1.Direct measurement. 2. Temporal resolution state. 3. Special resolution The five key stages for producing resting state EEG findings include re-referencing, filtering, segment extraction, poor electrode interpolation, and artifact elimination using independent component analysis (ICA).EEGLAB tools describe how to accomplish things using colored arrows. ICLable provides automated independent component categorization when ICA is completed.On data segments containing continuous raw EEG signals that were characterized as a two-dimensional matrix (electrodes* time), they utilized four forms of filtering: high-pass, low-pass, bandpass, and notch filters. When the segment length is set to 2 seconds, the frequency resolution is $0.5 \mathrm{~Hz}$. This adds a third dimension to the data, transforming it into a three-dimensional matrix (electrodes* time* segments).The Fourier transform was then employed to do spectrum analysis.By inserting in the frequency bin and electrode of interest in the result visu- 
alization for spectral analysis, the PSD (or magnitude, power) of an electrode may be determined.The variable is represented by the $\mathrm{x}$ axis, while the spectral variable is shown by the y axis.In a graph, they paid particular attention to the PSD scale. The linear scale $(\mathrm{mV} 2 / \mathrm{Hz})$ and the logarithmic scale $(\mathrm{mV} 2 / \mathrm{Hz})$ are two scales that show various features of the data and should be used in conjunction with our visualization purpose.After that, they do Connectivity and Micro state Analysis, as well as a minor bit of Advanced Analysis. One example of such a methodology is complex networks. To conclude, the Fourier transform generates data for connection measurements such as coherence, PLV, PLI, and wPLI by extracting frequency domain measurements such as magnitude, power, and PSD. GFP, GMD, and spatial correlation are all acronyms for the same thing, and GEV are used to cluster topographical maps and create micro states thanks to the spatial distribution of signals around the scalp and Due to the geographical dispersion of signals around the scalp, and GEV are utilized to cluster topographical maps and construct micro states.Using these approaches, we may extract relevant electrophysiological measurements that can be associated with certain psychological processes in a given setting. [80].

In another work, linear and nonlinear features were employed to extract EEG parameters after prepossessing the data. The characteristics of the EEG signal were extracted using wavelet coefficients and chaotic invariants such as Higuchi's algorithm's fractal dimension and correlation dimension, which revealed that in two emotional states, the Elman categorization accuracy was 82.7 percent. The aim of the study was to create a multi-modal connection between psycho-physiological waves and EEG in order to detect emotions. The device was created with the aim of recognizing emotion in offline EEG signals. They used Elman to examine the identification looking at how multi-modal bio-signals helped them recognize two emotional states. The method of labeling EEG signals is divided into three stages: self-evaluation, qualitative analysis, and quantitative. They used the Elman network to classify the data which is a two-layer back propagation neural network with feedback link between the hidden layer's output and its input. Elman network can learn to recognize and produce both temporal and spatial patterns thanks to this feedback route. As a result, they used approximately 65 percent of the data for training, 25 percent for checking whether the learnt link between EEG signals and emotion is right, and the remaining $10 \%$ for data validation. Using EEG signals, we achieved the highest accuracy of 82.7 percent for the two groups (calm vs. pessimistic exited)[23].

Another study focused on estimating students39; cognitive load using EEG. They discovered that task complexity had a main impact on estimates. EEG was found to be a reliable data source for modeling learners state of mental over the course of a 90-minute session. Their project39; s overall aim was to create a device that could quantify cognitive load. They made 24 dry electrodes in a headset that fit comfortably on students39; heads. In comparison to the more time-consuming set-up procedures used previously, using dry electrodes made collecting EEG in ecological contexts much more feasible. Furthermore, students were relatively unrestricted in their movements and could walk about freely in their chairs [55]. There was another paper which talked about not only the cognitive load, but also valence state of the brain. The goal of the paper was not to minimize the cognitive load but to optimize 
it. The novelty of the paper was in the methodology which was comparing CLI of the first channel with all other channels and It was used to evaluate a learning environment's efficiency as determined by changes in brain activity during learning scenarios. The article included three types of cognitive load measures: 1) subjective assessment based on felt mental burden, 2) objective assessment based on perceived mental stress, and 3) objective assessment based on perceived mental stress. 2. Measurement of performance- the result of a defined scale of regular task performance and 3. Psycho-physiological measures. The most effective EEG analysis was power spectrum analysis, which allowed them to see how synchronously the neurons that generate the EEG output oscillate at different frequencies. Theta-Alpha channel EEG measurements in frontal and parietal brain areas were shown to correspond with mental/cognitive load. The majority of research have shown that frontal Theta rises $[52]$.

As a contrast - enhanced hallmark, employ the Largest Lyapunov Exponent (LLE), Higuchi Fractal Dimension (HFD), and Sample Entropy (SampEn) of mental load processing to investigate how the brain's complexity varies throughout cognitive loading. In a publication, the suggested technique proved combined Under four different types of Cognitive Tasks (Emotion, Focus, Memory, and Problem Solving), four participants were given time-space representations of different Brain Arpeggios. With a $99 \%$ accuracy, these metrics were able to distinguish between distinct cognitive states such as emotion, concentration, memory, and problem solving. In signal processing, However, the elimination of eye blink and muscular artefacts from EEG data requires principal component analysis or independent component analysis (PCA/ ICA), however Getting rid of eye blink and muscular artefacts without jeopardizing the core EEG patterns is still a major problem. As a result, applying PCA/ICA-based noise reduction algorithms directly is not recommended.

In that work, they directly observed and used Dynamical Wavelet Compromise capabilities to safeguard just those parts of the EEG signal that correspond to the frequencies required to distinguish the progressions caused by mental piling. The sign is DE noised utilizing wavelet parcel decay (WPD) strategy to lessen the antiquities and prepare the sign for Rhythms that are explicitly extracted. In this paper, they performed lots of data analysis process like Chaos Analysis, Fractal Analysis, Entropy Analysis, Statistical Significance Analysis for finding better accuracy. Based on supervised learning with nonlinear features, they examined the Support Vector Machine, Random Forest, and Decision Tree classification models for classification. The correlation of execution measurements of characterization models SVM, arbitrary backwoods and choice tree classifier is appeared number of bogus discoveries if there should be an occurrence of Random Forest is a lot of reduced than the order models of SVM and Decision Tree. In the case of the Random Forest classifier model, the unwavering quality score of $99.69 \%$ is also greater. As a result, we may conclude that the Random woodland order model is well-suited to predicting the kind of conceptual cycle based on nonlinear features extracted from EEG data. They furnish with the overall importance and method of deciphering the three distinctive Parameters that are non-linear and were chosen as part of our inquiry. It would assist the reader in comprehending the storylines and drawing appropriate translations. After evaluating, they discovered that issue solving is a 
high-demand task., in like manner some spatial and transient varieties are noticed. Beta action from the lower temporal and parietal region has massive high repetition movements that do not alter over time. For emotion recognition, the minimal theta movement is seen in the event of Temporal, Parietal and Central projections. The complexity of beta, alpha, and delta action varies greatly. There is an increase in complexity during knowledge preparation of the mind while doing the memory recognition process, which decreases during the fourth and fifth time windows. Entropy is observed to be lower than the previous state once the job is completed in the seventh time frame, supporting routineness, decrease in vulnerability of forecast [88].

The cognitive burden for building an intelligent guidance system for visually impaired individuals (VIPs) traversing new interior areas was investigated in a research. For this work they tried to implement signal processing and machine learning technique. The main purpose of the project was to assess cognitive load, with an emphasis on working memory stress, in order to develop a sophisticated motion system for people with vision disabilities to utilize for navigation. Subjective evaluations, task-invoked pupillary response EEG data, and functional magnetic resonance imaging were all used by the researchers to quantify cognitive load (fMRI). Increased cognitive burden is caused by distraction. The article analyzed various related publications, the majority of which were based on s root mean square value, sub band energy, power spectral density, and engagement index, and then utilized a neural network to characterize the workload, which was determined by identifying alpha and beta band events in the cerebellum's frontal, temporal, and front-central sections, alpha power dispersion between Workload (WKL) and TOT settings, and a decrease in WKL level discriminability by increasing TOT in both figures of statistical modifications in band power and cataloguing act, and so on. For the study of cognitive stress, they are seeking to add an extracted entropy characteristic based on frequency domain analysis. They used five-fold cross validation for calcification in their situation. Before averaging the results, one set is chosen at random and set aside for testing, while the remaining $\mathrm{N}-1$ is used for training. To create an optimal model of the SVM classifier, five-fold cross validation is used to the training set. N-1 signifies that $80 \%$ of the training dataset is chosen at random to build the SVM model, while the remaining $20 \%$ is observed to fit the model. They were able to extract Entropy characteristics from EEG recordings utilizing 16 electrodes and various bands by doing these analyses. To analyze and quantify the performance, performance criteria such as sensitivity, specificity, and accuracy were used [89].

Another paper discussed about predicting the cognitive workload using EEG during intelligence test. They applied a well-known progressive metrics test (an ideal framework where task difficulty gradually increases). The innovation is that, unlike generic techniques, our framework can accurately predict cognitive load using spectral data even for a small number of channels while generating an individually adjusted neural network for each participant. Previous studies have discrete levels (2,3 levels) for measuring workload, here they used a continuous scale for workload. The beta: alpha was used as an indicator. For CNA (central neural activity) they used the Lempel Ziv complexity (features like PS metrics, neural complexity metrics, connectivity metrics), multiscale entropy, detrended fluctuation analysis. Classifier like$\mathrm{RF}$, bagging Decision tree, XGboost algorithm. The best result was obtained using 
the XGBoost classifier with a step size of 0.05. The $\mathrm{r} 2$ was used as the primary indicator of model performance. [69].

Rab Nawaz [84] used a three-dimensional model of emotions called arousal, valence, and dominance. EEG was recorded during a video of one minute which was shown to the participants. They focused on discovering the characteristics to distinguish between emotions by analysing Entropy, fractal dimension, and statistical EEG data for characteristics and wavelet energy from which finest features are determined. The classification accuracy of their system using all characteristics in the statistical category for arousal is $78.96 \%$, for valence that is $77.62 \%$ and lastly $77.60 \%$ overall accuracy is obtained for dominance. Their findings showed that EEG's time domain property may effectively distinguish between various emotive states of mind. In addition, employing a three-dimensional emotion model enables for the categorization of similar feelings that were previously misclassified using a two-dimensional model. The findings of this research can be utilized to help construct emotion identification systems on the basis of EEG real time data. They compared feature extraction approaches utilizing three distinct machine learning classifiers in a quantitative study. They narrowed down the feature space using the feature selection methodology to prevent over-specification with a bigger amount of retrieved features. Consequently, they used PCA FS to identify the most important features and analyze which sort of feature is the most favorable. The outcomes revealed because statistical characteristics are the most sensitive characteristics measure for describing the emotional dynamics of the brain. They assessed the features' dependability by utilizing all available data features. After that they chose to work with halves with better characteristics of each category. Statistical features outscored the other feature categories in circumstances. From this they infer that in determining classification of state of emotion, EEG time domain statistics play a big part. Furthermore, utilizing PCA FS to reduce the feature space aids in the development of a quick emotion identification system without severely impacting the system's performance. The current study's findings provide researchers with a thorough overview of the most important elements to extract state of emotion from EEG.

Ricardo Ramos [85] states that how the system performs is influenced by the steps of extracting features, which aims to identify important patterns connected to various mental activities. As a result, a technique based on spectrogram is suggested in this paper to extract characteristics from EEG signals. To begin, STFT is implemented in EEG in order to produce representations of time frequency where the parameter used is window length reliant on frequency of the EEG signal. Spectral peaks are determined so that it can be utilized as a reference for the purpose of acquiring spectrogram. To get features from EEG three methods used are done on the basis of frequency and surfaces, local ternary pattern adaptation and the third is based on maximum peaks. A multi layer perceptron, SVM and k-nearest neighbors are utilized to assess the extracted descriptors. The suggested method was tested using a dataset from Bonn University, with the main aim of distinguishing a healthy individual and an epileptic episode class. According to the findings of the experiments, the suggested technique achieves accuracy of nearly hundred percent in numerous circumstances considering less characteristics than other comparable research. The effects of window type, length, and overlap on the STFT's resolution 
were taken into consideration. The characteristics of this proposal are based on these characteristics, which allow for increased energy in the spectral peaks. With fewer characteristics, energy, K means and LTP performed well which is 99.5 percent. Sengür et al. were able to achieve $100 \%$ categorization accuracy. Other ways to extract features from spectrogram EEGs have been proposed in the literature. They needed only three features to reach a good accuracy whereas others usually need four. The findings, on the contrary, demonstrate that a binary classification is the most relevant. The approach demonstrated in this study can be utilized as tool for identifying an epileptic attack, or it may be used to replicate or strengthen the visual analysis that is often offered, with the support of professionals analyzing the EEG data. The concept may be used to EEG devices in a clinic to enhance performance, with the time monitoring determined solely by physician judgment.

Milena Cukic [77] worked out that for effective therapy and to avoid tragic results, a reliable diagnosis of depressive illness is required. The main focus of this study was the performance of Higuchi's Fractal Dimension and Sample Entrop on EEG in diagnosing psychological illnesses. Both non-linear measures were shown to be able to distinguish EEG signals from patients and healthy control participants in this paper, confirming previous findings. The findings demonstrate that even with a minimal number of primary components, effective classification may be achieved. Classifier accuracy varied from 90.24 to 97.56 percent on average. SampEn performed better than the other two metrics. If any approach for extracting feature produces excellent classification accuracy independent of the machine learning approach used, this proves that the feature extraction approach is effective. These findings inspire more research with bigger sample sizes in the hopes of finding a diagnostic use in clinical medicine and psychiatry.

Liwei Cheng [76] assirted that the subjective motor intention is reflected by the motor imagery electroencephalography which is in short MI-EEG. It is gaining popularity in rehabilitation. The key to MI-effective EEG's application is knowing how to extract its characteristics properly and rapidly. An approach based on PCA and DBN, together called PCA-DBN, is presented depending on the examination and distinction of current feature extraction techniques. They achieved accuracy of 96.25 percent and 91.71 percent respectively while classifying BCI Competition 2,3 and 4, afterwards kappa value accuracy of 0.925 and 0.8342 after finishing task classification with the softmax classifier is got. The verification findings are subjected to a pair of sample tests where FDR is adjusted, and a comparison to other better classification algorithms revealed that the method outperforms them. Lastly, this approach is applied to extract laboratory data characteristics which gave $97.69 \%$ classification accuracy and a value of Kappa 0.9538. To address the issue of high variability, value distinction, and unnecessary data of MI-EEG eigenvalues are produced using standard feature extraction techniques. Deep learning methods are integrated with classical feature extraction techniques in this study, resulting in PCA-DBN, a MI-EEG approach for extraction of features on the basis of PCA and DBN. This technique successfully merges PCA and DBN to perform feature dimensionality reduction of EEG signals. In contrast to previous feature extraction methods, this technique obtains good results in measuring features of MI-EEG along with improvement in kappa value and classification accuracy. 


\section{Chapter 3}

\section{Background studies}

\subsection{EEG Signals}

Our brain is a part of the primary sensory system that is located in the skull and regulates a person's cognitive thought cycles and real activity (Gulli et al., 2003). The cerebrum, together with the spinal line and nerve organization, regulates the data stream across the body, as well as purposeful actions such as studying and talking, as well as mandatory reactions such as breathing and absorption (Gulli et al., 2003). Brain has electrical voltage which is known as brain wives. In the modern age now we can detect and record these electrical wives which are known as EEG signals. The full form of EEG is an electroencephalogram which can detect and record the activity of the brain from our scalp. It represents cortical electrical activity. Proper measurement of EEG signal is needed for good analysis. Moreover, identifying proper location is also important.

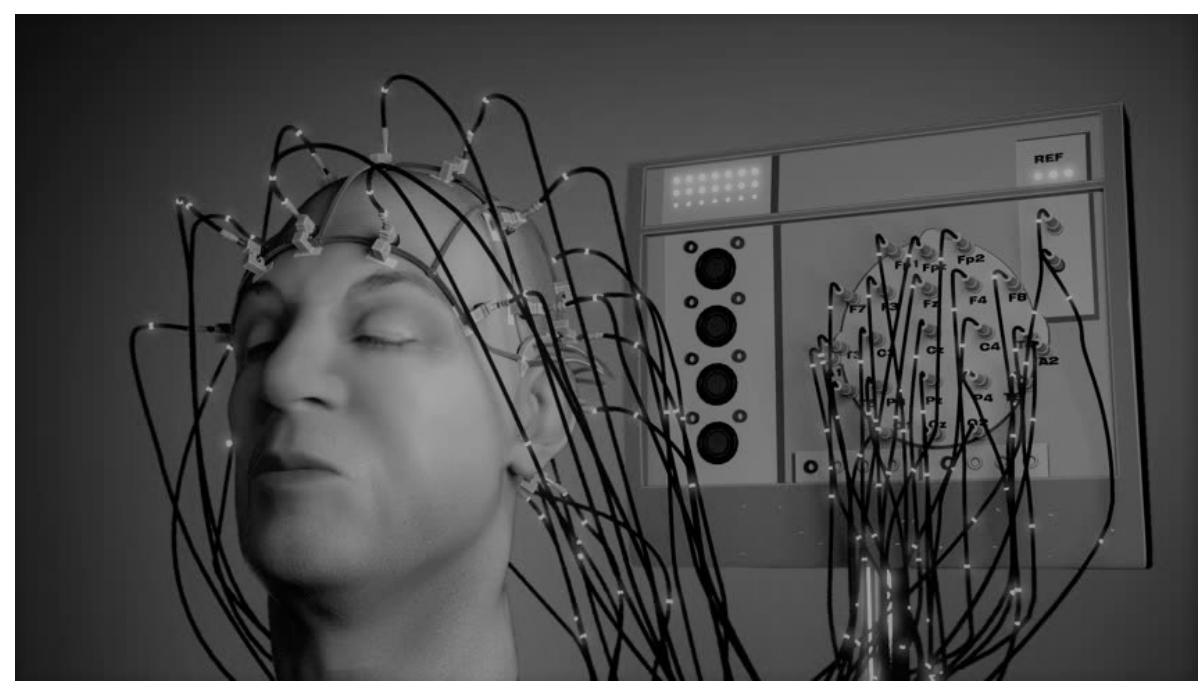

Figure 3.1: EEG Processing

This waves is highly small therefore micro volt is needed for measuring it. The most significant part of evaluation of EEG is frequency. The main frequencies of a human are Gamma, Beta, Alpha, Theta, and Delta. They have different frequencies of band waves. For example alpha has $8-12.5 \mathrm{~Hz}$, beta $12.5-29.5 \mathrm{~Hz}$, delta 0.5$4 \mathrm{~Hz}$, gamma is bigger $30 \mathrm{~Hz}$, theta $4-8 \mathrm{~Hz}$. The difference of frequency has a different meaning[44][60]. For measuring brain electrical movement we need a EEG 
machine which can detect and record frequency bands. Now-a-days it is useful for various researches like medical and microbiological research. The EEG machine has several elements like amplifiers, computer control modules, electrode and a monitor device[65]. (see in fig 3.1).

\subsection{CLI}

Though by the later 2000 the CLT was established well enough, the knowledge hunt for CLI or Cognitive Load Index was still in pursuit.This is the indicator of the cognitive load that how much work load or mental pressure a person is experiencing at that given particular time.Paas er al argued that combined with a perceived task difficulty, cognitive load rating can be done.If the same content is rated as more or less by the learner, depending on the form of its presentation,then this would serve as an additional indicator of the extraneous load concept of the CLT.Learning outcome,task complexity and objective measurements, all these happen to be in some sort indicators of cognitive load.The Event Related Desynchronisation/synchronisation (ERD/ERS) is an well established index for CLI[20] Sensorimotor ERD in the alpha and beta frequency bands $(8-13 \mathrm{~Hz}$ for mu rhythm and 15-25 Hz for beta rhythm) in humans may indicate brain activity similar to the monkey's mirror neuron system.[2]. The formula is represented by

$$
\Upsilon=\frac{\rho_{b}-\rho_{t}}{\rho b} * 100
$$

here $\Upsilon$ is an indicator of the cognitive load index and $b$ is a band power baseline interval and $t$ is a band power test interval.Another index called the TAR index was used for every analyzing windows of the EEG signal to as an index to the cognitive load.It was meaned for the whole EEG data segment that used to enclose them.Some light-based physiological measurements offer an indicator of neuronal activity that is directly related to a person's perceptual, cognitive, effective, or motor performance [8].

\subsection{Brain Waves}

The most valuable and significant part of human body is a brain. The approximate weight of brain is for adult human early $1.55 \mathrm{~kg}$ (3.38lb). Men weigh around 48.3606 ounce on average, whereas women weigh around 42.36403 ounce. The main function of the brain is passing message to all over the body with in very short time with the help of nerves. The main part of a nerve cell is neuron which is made by ((axons and dendrites). All neurons are connected to each others. These neurons are locked in through neurotransmitters. Here, a couple of nerves are straightforwardly associated with the cerebrum. Others had the chance to arrive at the mind through such a the spinal line, a control line that runs down the back. This spinal line connects to the brain, forming the Central Nervous System [65]. Brain controls our all organ. people call it mind. However, The language of computer science it calls processor who can process all require decisions for a body. In our body we have some sensory organ which pass the information in our brain like for visual organ (eyes), auditory organ (ears), skin(touch) etc. From taking all information brain can 
analyze it process it and make a reaction to it [54]. For visual impaired people they have different scenario. Their brain cannot get information from their visual organ (eyes). According to Steven Feldon, our brain deals one third works by the using of our visual system. Nonetheless, the brain evolves throughout time and receives information through its various physical organs. According to UCLA Department of Neurology researchers, visual impairment causes the human mind to undergo certain fundamental changes, demonstrating that the brain has the ability to reconstruct itself in response to a loss of tactile information [65]. However, Observing the brain movement to plan future man-machine interfaces is the point of Brain Computer Interfaces (BCI). A BCI may screen brain movement through an assortment of strategies, which can be coarsely named obtrusive and non-intrusive. Given the dangers produced by perpetual carefully embedded gadgets in the brain, and our concerns, we focus just on non-intrusive methodologies, specifically electrical mind signals as estimated by electroencephalogram (EEG)[15].

\subsubsection{Gamma waves}

Gamma waves has small amplitude as well this waves has to create very fast then others. We can differentiate two was for gamma waves. One is low gamma waves which has low frequency rate $(30-60) \mathrm{Hz}$. On the other hand high gamma waves has (60-200)Hz frequency rate [49]. Gamma waves are significant for learning, memory and data preparing. Gamma waves is able to influence the whole brain. They likewise put incredible effect upon complex cognitive capacities [45].

\subsubsection{Beta waves}

Beta waves are dynamic in a waking state. This recurrence is apparent in intelligent logical thinking[60]. In their action, we center on a critical thinking. When we have a lot of beta in our brain, we may experience excessive pressure and stress. When you consume coffee or another energizer, your beta action will typically rise[44]. This time people were more focused then others [48]. The result of too much beta waves create anxiety, stress. Less beta waves, on the other hand, are linked to ADHD. Depression and poor judgment are two symptoms of destitute cognition [3].

\subsubsection{Alpha waves}

The alpha waves create gap between our thinking and subconscious mind. It helps us to quiet of our behavior or it represent a feeling of relaxation[60]. Moreover, alpha is the recurrence range among beta and theta. On the off chance that we become pushed, a wonder called "alpha blocking" may happen which includes extreme beta movement and almost no alpha [44]. There have some problems when the alpha wave goes to low, as a result people suffer from anxiety, insomnia and stress full life. However, high range of alpha wave brings daydreaming. Most of the time alpha waves are found from adult person. For example after finishing any task then people take test which is result of alpha wave. Peace full workout like meditation is also result of alpha waves [65]. There have some investigations of EEG detailed with respect to the shortfall of alpha wave in blind subjects. The vast majority of the examinations show that the alpha movement is decreased or in any event, missing 
in blind subjects. However, some research provide us except for age, that EEG's of majority of normal visually impaired children provide normal alpha behavior [59].

\subsubsection{Theta waves}

This is highly low frequency wave. Mainly generated highly deep and row nature. Theta waves are engaged with rest or daydreaming. This brain wave can demonstrate instinct or automatic task [60]. Moreover, Ideal outcome is making enthusiastic, instinct.When theta waves increase, it generates hyperactivity and makes it unable to think clearly. Additionally, this signal is responsible for undermining attempts and may cause depression. They are in an unexpected way associated with task trouble. Increment level of theta waves can cause higher undertaking challenges [65].Furthermore, Theta waves are linked to our discovering and experiencing powerful and deep emotions. Because they are in a deeply relaxed, semi-hypnotic state, a lot of theta activation may make them more prone to depressive episodes and make them "exceptionally suggestible." It is additionally engaged with therapeutic rest [44].

\subsubsection{Delta waves}

Delta waves happen during contemplation in a condition of profound rest or extreme lethargy like coma. In cases of cerebrum damage, unusual delta activity may occur if the individual has learning disabilities or has difficulty maintaining aware consciousness[60]. Furthermore, this is the slowest measured brain signal in humans. They are typically discovered in both newborns and little children. They've also been linked to oblivious genuine abilities, like regulating heartbeat and digestion. After a good night's sleep, a sufficient amount of delta waves is produced, which helps us feel completely recharged [44].

\subsection{Wavelet Transform}

Wavelet has in reached past story. The principal writing that released with the wavelet transform is Haar wavelet. The mathematician Alfrd Haar in 1909 first time proposed it. Nonetheless, the idea of the wavelet didn't exist around then. Until 1981, the idea was proposed by the geophysicist Jean Morlet [22].In comparison to the STFT, the wavelet transform (WT) is a mapping from one dimension to another $L^{2}(R) \rightarrow L^{2}\left(R^{2}\right)$ with superior time-frequency specificity. Wavelet is a powerful time/frequency investigation apparatus for examining transient signs. Its element extraction and express characteristics may be used to investigate a variety of biological signal transient events. Because of its attractive qualities, the wavelet change is an effective signal handling equipment.,Localization of time-frequency, for example (obtaining a signal at a particular time - frequency, or features are extracted at different scales in diverse locations of area) as well as multi-rate filtering (dividing signs with various frequencies) (Meyer, 1993; Burrus et al., 1998; Rao also, Bopardikar 1998; Rao also, Bopardikar 1998; Rao also, Bopardikar 1998; Rao also, Bopardikar 1998; Rao also, Bopardikar; Samant and Adeli, 2000) [6]. There are two types of wavelet transforms: continuous wavelet transform (CWT) and discrete wavelet transform (DWT). 


\subsubsection{DWT $(\mathrm{db} 4)$}

Discrete Wavelet Transform is a fantastic frequency tool for time. It is widely used in PC-assisted signal examination of epileptic electroencephalography (EEG), such as seizure detection, throughout the last decade.In our method, DWT played a massive part. A wavelet is a rapidly disappearing oscillating function in temporal and frequency domains. The signal is split into scaled and translated versions $(\psi a, b(t))$ of a single $\psi(t)$ termed mother wavelet in wavelet based analysis.

$$
\psi_{m, n}(y)=\frac{1}{\sqrt{|m|}} \psi\left(\frac{y-n}{m}\right)
$$

where the scale and translation parameters are $\mathrm{m}$ and $\mathrm{n}$, respectively, and $\mathrm{m} 0$. By discretizing the parameters $\mathrm{m}$ and $\mathrm{n}$, the discrete wavelet transform (DWT) was produced. The DWT uses dyadic sampling with parameters $m$ and $n$ based on powers of two $m=2^{x}$ and $n=k 2^{x}$ with $x, k \in Z$ in its most common version, We get the dyadic wavelets by inserting in Eq 3.3 [51]:

$$
\psi_{x, k}(y)=2^{-x / 2} \psi\left(2^{-x} y-k\right)
$$

Of note, DWT could be written as

$$
d_{x, k}=\int_{-\infty}^{+\infty} s(y) 2^{-x / 2} \psi^{*}\left(2^{-x} y-k\right) \mathrm{d} t=\left\langle s(y), \psi_{x, k}(y)\right\rangle
$$

Wavelet coefficients at level $\mathrm{x}$ and location $\mathrm{k}$ are known as $\mathrm{dx}, \mathrm{k}$. In seizure detection, these coefficients were utilized to build the feature vector of each EEG segment [51].

\subsection{Fourier Transform}

The Fourier Series is a mathematical concept that can be applied to virtually anything in our everyday lives. The Fourier Transform enables one to see these wave forms in a more efficient way.Separating the blended paint is equivalent to the Fourier transform. The formula is the Fourier transform, a list of ingredients and how to add them, if the product is the waveform. Separating the signal from the noise in data obtained for astronomy, medicine, biology, and chemistry are some of its main applications.

\subsubsection{FFT}

The fast Fourier transform (FFT) approach analyzes EEG data using statistical means or software. The power spectral density (PSD) computation is used to determine attributes of the acquired EEG signal to be evaluated in order to selectively reflect the EEG samples signal.The major feature wave forms of the EEG spectrum are found in four frequencies[36].The PSD is produced by Fourier processing a nonparametrically recorded auto correlation series. Welch's method is one of them. When the data sequence is subjected to data windowing, modified periodograms result.

The data sequence $x_{i}(p)$ is written as $x_{i}(p)$. 


$$
\begin{aligned}
x_{i}(p)=x(p+i D), \quad p & =0,1,2, \ldots, M-1 \\
\text { while } i & =0,1,2, \ldots, L-1
\end{aligned}
$$

Take $i D$ as the ith sequence's start point. The data sections then are represented by $L$ of length $2 M$. The performance periodograms results are as follows:

$$
\stackrel{z}{P}_{x x}^{(i)}(f)=\frac{1}{M U}\left|\sum_{p=0}^{M-1} x_{i}(p) w(p) e^{-j 2 \pi f p}\right|^{2}
$$

And in window function, $U$ represents the power's normalization factor and is selected in such a way that

$$
U=\frac{1}{M} \sum_{p=0}^{M-1} w^{2}(p)
$$

where the window function is $w(p)$. Welch's power range is calculated as the average of these adjusted periodograms:

$$
P_{x x}^{W}=\frac{1}{L} \sum_{i=0}^{L-1} \widetilde{\widetilde{P}}_{x x}^{(i)}(f)
$$

\subsubsection{DFT}

Fourier analysis is required for data processing since it divides a signal into constituent sinusoids of different frequency. When doing Fourier analysis on sampled vector data, the discrete Fourier transform is utilized (DFT). The fast Fourier transform (FFT) is a non-separate technique for determining the DFT of a sequence[29]. This is extremely effective in signal processing, where it can be used for anything from filtering to frequency analysis to power spectrum measurement . Each level's calculation using DFT provides an idea of the frequencies found in the bands. For intermittent, discrete-time, or digital signals, and DFT is used signal.

$$
X[k]=\sum_{p=0}^{N-1} x[p] e^{-j \frac{2 \pi k}{N} p} .
$$

According to these equations, the coefficients $\mathrm{X}[\mathrm{k}]$ view a discrete-time signal that is periodicx $[\mathrm{p}]$. Notice, that only $\mathrm{N}$ samples of the time signal $x[p]$ are used to compute $X[k]$ (for $k=-\infty . . \infty$ ), and also only $\mathrm{N}$ of the coefficients $\mathrm{X}[\mathrm{k}] \mathrm{In}$ the inverse transform, are used.The spectrum of a periodic discrete-time signal is continuous (just like any other discrete time signal), i.e., $\mathrm{X}[\mathrm{k}+\mathrm{N}]=\mathrm{X}[\mathrm{k}]$. The $\mathrm{X}[\mathrm{k}]$ terms are evenly spaced samples of the continuous spectrum $\mathrm{X} 1(\mathrm{ej})$ of the signal $\mathrm{x} I[\mathrm{p}]=\mathrm{x}[\mathrm{p}]$ for $0 \leq \mathrm{n}<\mathrm{N} ; \mathrm{xI}[\mathrm{p}]=0$ for $p<0, p \geq N$

\subsubsection{IFFT}

$$
X(p)=\frac{1}{N} * \sum_{k=0}^{N-1} x(k) * e^{i * 2 * p i^{*} p^{*} k / N} \ldots \text { IFFT Equation }
$$


Where,

$$
\begin{aligned}
& \mathrm{X}(\mathrm{k}) \rightarrow \text { Frequency Domain Samples } \\
& \mathrm{X}(\mathrm{p})->\text { Time Domain Samples } \\
& \mathrm{N} \rightarrow \text { Fast Fourier Transform Size } \\
& \mathrm{k} \rightarrow 0,1,2, \ldots . ., \mathrm{N}-1
\end{aligned}
$$

The Inverse Fast Fourier Transform (IFFT) is the abbreviation for Inverse Fast Fourier Transform (Inverse Fast Fourier Transform).IFFT is a technique for converting a frequency domain vector signal to a time domain vector signal. It occurs after the modulator block in an OFDM transmitter.

\subsection{Machine Learning:}

Machine learning (ML) is a technology that gives AI software ability to improve prediction accuracy without having to be explicitly trained.The main objective is for computers to learn on their own, without the need for human interference, and to adapt their behaviour accordingly.In order to simulate new output values, machine learning algorithms use past data as input. Building a machine learning program can be broken down into four simple stages (or model). These are usually carried out by data scientists in collaboration with the industry experts to whom the model is being built.To build a model, we must first pick and prepare a training data set and then train it for the model to be refined.It is commonly used in research, especially in the areas of EEG signal and cognitive load.

\section{Supervised Learning:}

Supervised machine learning is a subset of artificial intelligence and computer learning. It is defined by the use of named data sets to train algorithms that accurately interpret data or predict outcomes. As the model takes in the input data, a reinforcement learning algorithm updates the model's weights, indicating that the model is appropriately fitted.Companies may use supervised learning to solve a host of largescale problems, such as classification of spam.A training set is used to direct models to obtain the optimal outcomes of supervised learning. Since this training data set contains both correct and incorrect outputs, the model can learn over time. The algorithm's precision is assessed using the loss parameter, which is modified before the error is minimized. There are two kinds of supervised learning problems in data mining: classification and regression.In terms of grouping. An algorithm is used to classify test data and distribute it to unique classes. It looks for specific individuals in the data and tries to make inferences about how they should be classified or defined.On a slow decline For looking at the relationships between variables, regression can be used.It can also be used to forecast overall revenues for a company. Popular regression approaches include linear regression, logistic regression, and polynomial regression.

\subsubsection{Decision Tree}

By recursively constructing a tree from these subproblems, The decision tree acts as a supervised classification technique that separates a difficult problem into subproblems.Each leaf node in a decision tree is given a class name, and non terminal 
nodes, such as the root and internal nodes, have attribute checking criteria to identify records with specific properties.

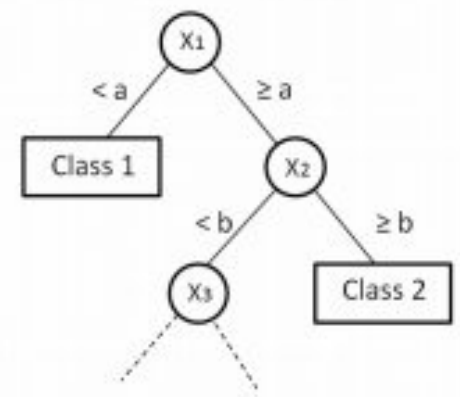

Figure 3.2: Decision tree example

A standard decision tree schema is shown IN Figure 3.2 The Y decision nodes are variables. The attribute boundaries, which divide the decision into three tree pathways, are indicated by variables a and b. These characteristics might be numerical or non-numerical. The tree-leaf-patterned class variables are in charge of identifying the object under study.[50].In the late $1970 \mathrm{~s}$ and early $1980 \mathrm{~s}$,. ID3, a decision tree-generating algorithm, was designed by J Ross Quinlan. After a few years, he introduced the C4.5 method, which is a more advanced variant of ID3. According to the authors, it serves as a foundation for current supervised techniques. Algorithm of the software J48 is a Java-based version of the C4.5 classification method. It was created as a result of the requirement to recode the algorithm., C4.5 was created using the mathrm $\mathrm{C}$ programming language. To increase the estimation capabilities of decision trees, this methodology employs the partition and conquer method.It typically employs the best assessed step locally, regardless of whether or not this step delivers the optimal solution, splits a problem into numerous subproblems by forming subtrees between the stem and leaves, creates subproblems to break an issue into many subproblems. break a problem into several subproblems by building subtrees between the root and the leaves of a tree by constructing subtrees between them, divide the problem into several subproblems When used on the test set of data,Classification algorithms look for models with the best accuracy and lowest error rate.

\subsubsection{SVM}

SVM is a real machine learning-based methodology underlying classifiers that is increasingly in the spotlight.[62] To categorize eyes blinking, a comparison was made between SVM and ANN (artificial neural network), with the SVM based tool demonstrating superior accuracy. The SVM method has lately been popular in EEG categorization for research into a number of brain illnesses, including Alzheimer's disease. The use of an SVM classifier for EEG data to identify the beginning of epileptic seizures is demonstrated.. The results were obtained using an RBF kernel that generated features for both seizure and non-seizure behavior.As observed in, the EEG classification may be used to diagnose brain tumors and identify tiredness while driving. To extract uniform properties from the data, the referred articles employed 
a spectrum analysis approach. Spectrum analysis, such as the FFT methodology, is incompatible with complex signals like EEG since it is dependent on basic functions (such as sinusoids). Using temporal frequency analysis and a continuous wavelet transform, we extract properties.which is the most comparable to non-stationary signals like the EEG, as well as an approach similar to that described in. The SVM model has also been used to characterize signals in schizophrenia disorders, with features derived using an autoregressive model (AR) to preprocess data and then classified using SVM. To reduce features (data preprocessing), linear dit regression was used (LDA). We used PCA for this because it has a higher reduction potential than LDA.

\subsubsection{Random Forest}

Random forest (RF), a well-known method developed by Leo Breiman in 2001, has been shown to work well in classification issues. RF creates an ensemble of classification trees using bagging and random variable selection based on a bootstrap sample of the data [35]. A variable candidate set is chosen at random from the whole variable set at each division. The splitter is partially assessed at random after each tree is grown on various random sub samples. Each tree is completely matured to obtain a minimal bias. Bagging and random variable selection preserve the low correlation for individual trees. The algorithm scales a large ensemble of low-bias, high-variance, low-correlation trees to produce an ensemble forest. As mentioned further down, a variety of algorithmic difficulties were examined, including parameter optimization and the processing of unbalanced data sets.

$T$ : Training number of set $\left(\vec{x}_{1}, y_{1}\right),\left(\vec{x}_{2}, y_{1}\right), \ldots,\left(\vec{x}_{n}, y_{n}\right)$

$N_{\text {tree }}:$ trees number to be built;

$M_{t r y}$ : For each node, the number of variables chosen for splitting;

Training: each $b=1: N_{\text {tree }}$ Make a sample of bootstrapped data. $X_{b}$ from the collection of trainning provided $T$. At each tree node $t r b$, select $M_{t r y}$ random variables and try to figure out the optimal split between them $M_{\text {try }}$ variables.Using the bootstrapped examples above, create an unpruned tree trb. classification's end: Sort by who has the most votes among the $N$ trees.

$$
\begin{aligned}
& \text { Compute } \\
& f_{\text {avg }}(X):=\left(p_{1}(X), \ldots, p_{k}(X)\right):=\frac{1}{N} \sum_{b=1}^{N} f_{i}(X) \\
& \quad f_{R F}(X):=\operatorname{argmax}_{k}\left\{p_{1}(X), \ldots, p_{k}(X)\right\}
\end{aligned}
$$

\subsubsection{Gradient Boost}

Gradient boosting is one of the most widely used eeg signal classifiers. Since the features have been extracted, the EEG can be classified as[42] seizure or non-seizure. Gradient boosting was the classifier used in this study.We can find ensemble of classifiers after step $m$ by $F_{g}$, the training data by $X=\left\{x_{i} \in R^{K}, i=1, \cdots N\right\}$, and related class labels by $Y=\left\{y_{i} \in\{0,1\}, i=1, \cdots N\right\}$. 
the weak one least square regression, and the logistic regression model was as follows:

$$
p_{g}\left(y_{i}=1 \mid x_{i}\right)=\frac{e^{F_{g}\left(x_{i}\right)}}{e^{F_{g}\left(x_{1}\right)}+e^{-F_{g}\left(x_{i}\right)}}, i=1, \cdots N
$$

The Bernoulli log-likelihood of the logistic regression model is optimized via gradient boosting, with the Bernoulli log-likelihood of $F_{g}$ being stated as follows:

$$
L\left(F_{g}\right)=\log \left(\prod_{i=1}^{N} p_{g}\left(y_{i}=1 \mid x_{i}\right)^{y_{i}} p_{g}\left(y_{i}=0 \mid x_{i}\right)^{1-y_{1}}\right)
$$

The algorithm implementation is shown in below steps:

(1) Start with $F_{0}\left(x_{i}\right)=0, i=1, \cdots N$ and $p_{0}\left(y_{i}=1 \mid x_{i}\right)=0.5$.

(2) Repeat for $g=1,2, \ldots, G$ :

(a) Compute the gradient of the likelihood function with respect to $F=F_{g-1}$ : $\tilde{y}_{i}=2\left(y_{i}-p_{g-1}\left(y_{i}=1 \mid x_{i}\right)\right), \quad i=1,-N$

(b) A weak classifier $f_{g}$ with the best least squares match to the gradient is chosen: $f_{g}=\arg \min _{f} \sum_{i=1}^{N}\left(\tilde{y}_{i}-f\left(x_{i}\right)\right)^{2}$

(c) Determine the coefficient $\gamma_{g}: \gamma_{g}=\arg \max _{\gamma} L\left(F_{g-1}+\gamma f_{g}\right)$

(d) Update the classifier:

$F_{g}=F_{g-1}+\varepsilon \gamma_{g} f_{g}$ Here, $\gamma_{g}$ shrinks to a small value through multiplication with a small $\varepsilon$ at each stage to increase the boosting algorithm's generalization efficiency. In this paper, the value of $\varepsilon$ is given as 0.05 .

(e) evaluate the probability using Eq. 3.13.

Training and testing are the two steps of the classification process. The feature vectors developed for the training data set were fed into a gradient boosting classifier throughout each training stage.The training samples were then entered into the qualified gradient boosting classifier, which had a range of 0 to 1.The threshold value is chosen to decrease the number of categorized training samples, and it will be utilized in post-processing. The qualified classifier was used to the testing data in the testing stage, and the classifier's probabilities results were processed in the following manner.

\subsubsection{Naive Bayes}

Based on a set of training data, Naive Bayes is one of the finest classifiers for determining the optimal output hypothesis H.As can be seen, the Bayes theorem assists us in calculating the a posteriori probability of both the data on the hypothesis and the entire data [31]. The probability of a hypothesis that defines the relevance of a variable based on a priori probability on the likelihood of each hypothesis is known as a posteriori probability. 


$$
P\left(v_{j} \mid A\right)=\frac{P\left(k \mid v_{j}\right) \times P\left(v_{j}\right)}{P(k)}
$$

Here in the set of hypotheses $V$ the hypotheses is $v_{j}$ and $k$ is the set of attributes $\left\langle a_{1}, a_{2}, \ldots, a_{n}\right\rangle$ that explains the data When $k$ has multiple attributes, it is essential to analyse them. $P\left(a_{1}, a_{2}, \ldots, a_{n} \mid v_{j}\right)$ to calculate $P\left(v_{j} \mid a_{1}, a_{2}, \ldots, a_{n}\right)$. ths main problem is that to calculate $P\left(k \mid v_{j}\right)$ It is essential to capture a number of samples. Furthermore, it is computationally expensive since the joint odds for all possible $k$ can be determined. Naive Bayes classifier in response to this, which implies that all attributes in $A$ are independent. There is literature that discusses this premise and the good outcomes that have resulted from it. They say that even though these attributes are not completely independent, better classification efficiency can be achieved. As a result, the joint likelihood is derived .

$$
P\left(a_{1}, a_{2}, \ldots, a_{n} \mid v_{j}\right)=\prod_{i} P\left(a_{i} \mid m_{j}\right)
$$

And the output of the classifier is set by:

$$
v_{M A P}=\arg \max _{m_{j} \in m}\left\{P\left(m_{j}\right) \times \prod_{i} P\left(a_{i} \mid v_{j}\right)\right\}
$$

The total posteriori value determined within the space of hypotheses $m$ is $m_{M A P}$. It's worth noting that only measuring the probability distribution of each attribute for each class is needed; calculating $P(A)$ is not needed because it's a concept that applies to all classes and therefore isn't applicable classification .

\subsubsection{K-Nearest Neighbour}

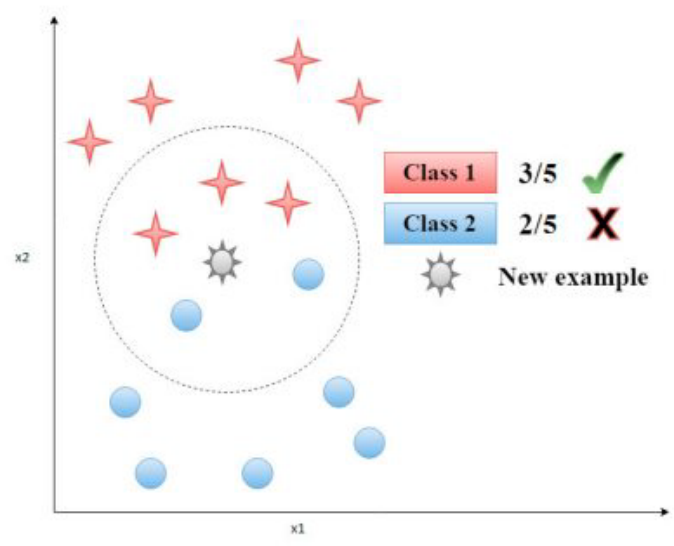

Figure 3.3: K-nearest neighbor

A non-parametric approach for classifying data objects according to their number of neighbors. [58]The KNN method works in two steps: first, it determines the number of nearest neighbors, and then it assigns the data item to one of many categories. As seen in equation 3.18, Euclidean distance use distance metrics to find the neighbor.

$$
\text { Distance }(\mathrm{x}, \mathrm{y})=\sqrt{\Sigma_{i}\left(x_{i}-y_{i}\right)^{2}}
$$


It chooses the k samples nearest to them from the training set., then asks their class for a plurality vote, where $k$ is supposed to be an odd number to prevent complexity. The architecture of the KNN classifier is seen in Figure 3.3. Class 1 and class 2 are the two types of classes. The first class is denoted by a red asterisk, and class 2 is marked by blue circles. The number $\mathrm{K}$ was selected, and three samples from class 1 and two samples from class 2 are some of the five closest neighbors. The KNN classifier works on the principle of allocating a new sample to the class with the most votes in the specified $\mathrm{K}$. As a result, the new test example is assigned to class 1 .

\subsubsection{Logistic Regression}

Logistic regression [10] is very popular and mostly used statistical modeling technique where the probability, $P_{1}$, is compared to a set of explanatory variables in the form

$$
\begin{aligned}
\operatorname{logit}\left(P_{1}\right) & =\ln \left(\frac{P_{1}}{1-P_{1}}\right)=\beta_{0}+\beta_{1} x_{1}+\cdots+\beta_{n} x_{n} \\
& =\beta_{0}+\sum_{i=1}^{n} \beta_{i} x_{i}
\end{aligned}
$$

In the Eq. 3.19, $\beta_{0}$ is the difference between and $\beta_{1}, \beta_{2}, \ldots, \beta_{n}$ are the coefficients that are linked to the explanatory variable $x_{1}, x_{2}, \ldots, x_{n}$. The average wavelet coefficients of provided -channel EEG recordings are used as input variables. There are only two conceivable interpretations for a binary attribute.such as yes/no, on/off, survive/die, or 1/0, which denote the occurrence or non-occurrence of a specific event .Here Continuous, dichotomous, discrete, or a mixture of explanatory variables can be used. Ordinary linear regression (OLR) with a binary outcome based on the least squares approach would provide nonsensical results. The response (dependent) variable, as in Eq.3.19, is the natural logarithm of the dependent variable, This is the possibility of a behavior occurring divided by the likelihood of it not occurring ( probability of being epileptic or not). Because it does not presume that the connection between the eigenvalues is linear, logistic regression has fewer constraints than OLR. Gaussian distributed independent variables are not necessary to explain the relation between the explanatory factors and the response variable. When compared to OLR,Logistic regression calculates changes in the response variable's logarithm of odds, whereas logistic regression examines differences in the response variable's logarithm of odds. The answer and independent variable do not have a linear connection when regressed since the explanatory factors and the odds logarithm are linearly connected. as described in Eq. 3.19, a function of the independent variable, the likelihood of an event occurring is nonlinear.

$$
P_{1}(x)=\frac{1}{1+\mathrm{e}^{-\log i t\left(P_{1}(x)\right)}}=\frac{1}{1+\mathrm{e}^{-\left(\beta_{0}+\sum_{i=1}^{n} \beta_{i} x_{i}\right)}}
$$

Logistic regression, unlike OLR, will determine the probability values. $\left(P_{1}\right)$ to lie between 0 and $1\left(P_{1} \rightarrow 0\right.$ as the right-hand side of Eq. 3.20 gives $-\infty$, and $P_{1} \rightarrow 1$ 
as it gives $+\infty$ ). The probability estimation (MLE) methodology is widely used to estimate the coefficients. $\beta_{0}, \beta_{1}, \ldots, \beta_{n}$ in the logistic regression equation This method differs from the traditional least squares method for calculating linear regression coefficients(OLS)[10]. The OLS method aims to reduce the number of squared distances between all data points and the regression line to the absolute minimum. The MLE method, on the other hand, aims to maximize log probability, which determines how likely (or how likely) it is to predict the observed values of the dependent variable from the observed values of the independent variables.Unlike the OLS approach, the MLE approach is a time-saving strategy that starts with an estimate of the regression equation coefficients before determining the location and degree of change in the coefficients that will improve the probability function.After determining the initial function, the residuals are assessed, and a new calculation of an improved function is generated. This test is carried out until a convergence requirement is satisfied (for example, the Wald test, log likelihood ratio test, classification tables, and so on). The coefficients were determined by reducing the log probabilistic model (using Newton's method), which is defined as the sum of the logarithms of the predicted probability of an event in cases where the event occurred and the logarithms of the predicted probabilities of non-occurrence in cases where the event did not occur. [10] .

\subsubsection{Stocastic gradient Descent}

Stocastic gradient Descent is a kind of supervised learning, in which each data point in the data collection is $z$ demonstrates a pair $(p, q)$ contains the input characteristics $p$ and scalar class labels $q$. An objective function is used here. $\mathcal{L}(\hat{q}, q)$ is a term used to describe the distinction between class labels predicted by a neural network and those viewed (considered as $\hat{q}$ ) and the actual class labels $q$. A family $\mathcal{F}$ of functions $f_{k}(p)$ is defined by a weight vector $k$. The function $f \in \mathcal{F}$ reduces the size of the error section $Q(z, k)=\mathcal{L}\left(f_{k}(p), q\right)$ This was calculated by averaging the training samples[87].

$$
E_{m}(f)=\frac{1}{m} \sum_{i=i}^{m} \mathcal{L}\left(f\left(p_{i}\right), q_{i}\right)
$$

The statistical risk $E_{m}(f)$ must be kept to a minimum. On the training package, it evaluates the model's efficacy.Statistical danger is most commonly the case $\left(E_{m}(f)\right)$ is minimised by the gradient descent(GD) approach and weights $k$ are updated depending on the statistical risk gradient $\left(E_{m}(f)\right)$. The weight-loss formula is as follows:

$$
k_{t+1}=k_{t}-\eta \frac{1}{m} \sum_{i=1}^{m} \nabla_{k} Q\left(z_{i}, k_{i}\right)
$$

where $\eta$ signifies the learning rate or phase scale In practice, the learning rate is commonly fixed between 0 and 1. A significant rate of learning was also observed by the researchers. $\eta$ creates too quick convergence, which leads to less experimentation and poor generalization.Small causes $\eta$ on the other hand, slows down the algorithm's convergence., implying further experimentation and strong generalization but less manipulation. As a result, in practice, researchers aim to strike a balance between experimentation and exploitation correctly setting learning rate $\eta$ 
The majority of the time, it is chosen by trials, but the range should be between 0 and 1. Gradient descent algorithm does not work effectively where the training data set is huge, . The Stochastic Gradient Descent (SGD) technique can solve this problem. The SGD algorithm is a stochastic variation of the GD method.The gradient of $E_{m}(f)$ in (SDG) are not computed accurately; instead, they are calculated based on a randomly picked single data sample. $D_{i}$ in each iteration:

$$
k_{t+1}=k_{t}-\eta_{t} \nabla_{k} Q\left(z_{i}, k_{i}\right)
$$

The stochastic method $\left\{k_{t}, t=1, \ldots ..\right\}$ where iteration is based on data samples that are picked at random.

\subsubsection{MLP}

The multi-layer perceptron is a type of artificial neural network that demonstrates how our brain's neurons function. This neural network learns from training data to be used. MLP, that has three layers, is used by the artificial neural network. Each layer has many nodes, and the neurons are replicated. The input layer, which comprises nodes that indicate the amount of features present in the training data collection, is the initial layer.Many secret layers make up the second layer. The amount of hidden layers in which they are contained are completely random. The output layer comes in third. The model of an artificial neural network may be shown in Figure 3.4 There is each layer of input, hidden and output layer in this image [34].Four nodes form the input layer. This is connected to the four nodes of the hidden layer, and the hidden layer nodes are connected to the output layer node.Weights represent the total of electrical impulses transmitted by connecting lines in neurons. Backpropagation learning is employed in this model to analyze and update the weights during the workout.The elements from each input node and the resulting weight are computed as the input for a transfer function at each node in the hidden layer which is activation function. The stimulation and regulation of electrical impulses are modeled using a transfer mechanism.

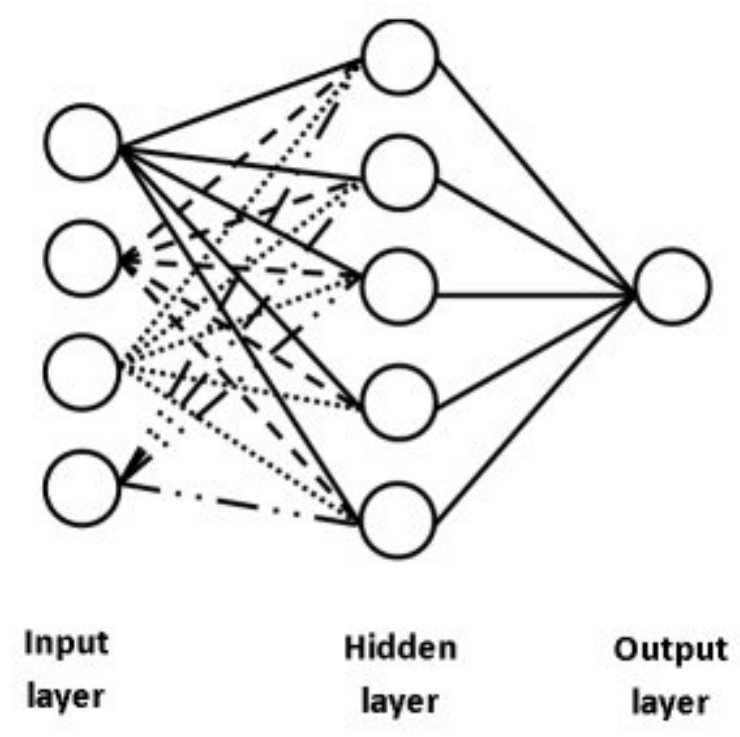

Figure 3.4: Multi layer Perception Layer 


\section{Chapter 4}

\section{Experimental setup}

Due to the outbreak of the COVID-19, we could not collect data on our own, so we worked with a very notable data set from the University of Iceland which was related to our topic. In the data set there was various forms of data among which we used the multi modal bio-signal data captured during different phase of mobility to check for the difference in cognitive load under different situation and detecting emotional states

\section{1 volunteers}

Nine healthy visually impaired volunteers were selected among which five was female(average age 42 years). All of the volunteers have different degrees of sight loss at different age of their lives. We divided the participants into 3 categories based on their sight loss. To make the situation easy for them, they were free to use their

\begin{tabular}{l|l} 
Category & Description \\
\hline VI-1 & vision less than 10 percent but more than 5 percent \\
VI-2 & vision less than 5 percent and little light perception \\
VI-3 & no light perception
\end{tabular}

Table 4.1: Category of volunteers

familiar Operations and Maintenance instructor if it would help them feel at ease. If they desired, they were also encouraged to stroll with their white canes. We selected them based on their free will and they were fit and healthy to perform various tasks that they were asked to do.All the data that we received was anonymized before analysis. They were told to avoid smoking regular or e-cigarettes, as well as caffeine and sweets, for around an hour before the walk. The study was approved by the National Bioethics Committee of Iceland.All of them shared the same experience that our given task was part of their day to day life as many of them were part-time job holder.

\subsection{The Indoor routes}

For the purpose of the study and for clear analysis, the route had to be set inside such a building that is not very intricate again sufficiently complex. After many 
observation the Haskolatorg building of the University of Iceland was selected. It was a multipurpose building that served various units for the university students like a bookstore, two restaurants, classrooms and reading rooms, the corridors and the interior of the building created a perfect combination of all the tasks a VIP could face in day to day life. With the help of the OM instructors, they planned a route to take the VIP thought the charted route pre-designed by the instructors and through circumstances where different levels of cognitive load likely to occur.Each participants walked the indoor route three times for training purpose on which they were guided fully of their first trial by their instructor then the following two times, it was just like the real life scenario, where they has to chart and navigate the whole route all by themselves. The following five tasks were the primary action or challenges for the VIP.

\begin{tabular}{||cr||}
\hline Indoor Route & Challenges \\
\hline \hline Automated Door & Find push button, enter the rotating door,avoid people \\
\hline Elevator & Travel between floors, getting in and out \\
\hline Narrow Corridor & avoiding people collision,noise \\
\hline Open Space & avoid table,chair,pillars,people \\
\hline Using Stairs & Spiral stair to move between floors \\
\hline
\end{tabular}

Table 4.2: Indoor Mobility challenges

The course was around 200 meters long, and each participant took between 4 and 8 minutes to walk it. When asked later it was revealed that all the nine participants experienced the proper indoor environments they face in their daily life. Video and audio was recorded using the camera of a mobile phone to facilitate data annotations. At the conclusion of the three trials, each participant was instructed to vocally describe where they felt cognitive burden.

\subsection{Data Acquisition}

As EEG is a visual analysis, the noise around the environment must be removed to get proper data. In this data set the Emotiv EMPOC+ device was used.It had 9 axis motion sensors.EPOC+'s EEG signal quality is statistically comparable to that of typical research grade devices that cost tens of thousands of dollars. This clearly states the cost effectiveness of the device.Registered of the 10-20 system locations, the device is a 16 dry-cell mobile headset, AF3,F7,F3,FC5,T7,P3(CMS) ,P7,O1,O2,P8,P4(DRL),T8,FC6,F4,F8,FC4, the sampling rate was set to $128 \mathrm{hz}$, because this does not affect the signal quality nor we will lose any important part of the Gamma rhythm, because for most clinical studies high gamma will extend to maximum $100 \mathrm{~Hz}$, Another reason to choose this device was It provides a very good performance-to-price ratio, which was the number os channels through EEG was recorded and the quality of the signals obtained and usability. By usability they meant the rehearsal time,the flexibility and the user's well being.

Another device, the Empatica E4 wristband was also used in the data set. The use of it was for measuring two things.BVP through a dorsal wrist photoplethysmography sensor $\left(\mathrm{fs}_{\mathrm{s}}=64 \mathrm{hz}\right)$ and EDA through 2 ventral wrist electrodes $\left(\mathrm{fs}_{\mathrm{s}}=4 \mathrm{hz}\right)$. The E4 

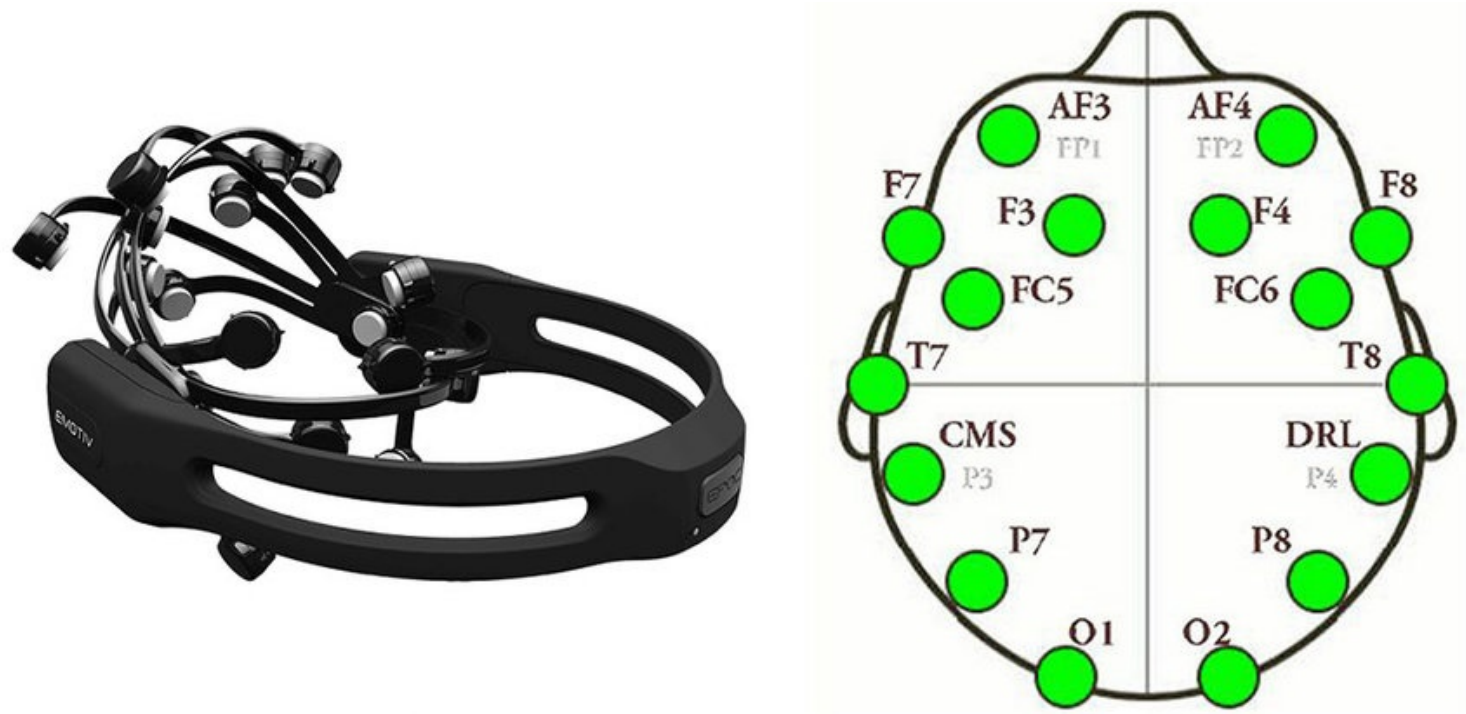

Figure 4.1: The Emotiv Epoc+ and the placement of electrodes

is presently the first commercially available multisensor device based on extensive scientific study in the fields of psycho physiology and psychological computing.It has a watch-like look and is cable-free. The gadget was originally placed on the VIP's non-dominant hand to reduce motion artifacts caused by holding the white cane. The original signal is divided up in frequencies using Fourier frequency analysis in order to eliminate certain frequencies before being transformed back into the signal with just the frequencies of interest. They mainly focused on the second trial's acquired data, because in the first trial there was some problem, there is another trail in the data set which is actually the average of the two trails. 


\section{Chapter 5}

\section{Methodology}

In this chapter there will be a brief and detailed discussion about our total system work flow and all the methodologies we used to conduct our research.It is very important to have a pre-planned methodology if someone wants to work with EEG signals. Though there are many form of methodologies regarding the detection of cognitive load,the need of measuring it objectively and validly has given rise to curiosity and has encouraged the exploration of many alternate methods[24].Applying a different methodology for the same research may lead to different outcomes and it is not possible in a single research to explore all the available methodologies of that particular fields[40]. In our methodology we tried to introduce a new concept with the old existing field of event related synchronization and de-synchronization by only passing the baseline power of the alpha band, but the ration of the AlphaBeta band and the ratio of the Alpha-Theta band which gave a much more efficient and perfect result for the classification.

\subsection{System Work Flow}

The structural middle ware and methodology is explained with the Fig 5.1 below.At First Raw signal is collected through the surface electrodes that was set down on the cranium by the use of the Emotiv epoch + headset positioning stratagem. Secondly initialisation procedure was conducted which was a combination of different band pass filters and setting the frequency to down sample and up sample the signals to eliminate the unwanted signals and to make the signal suitable for further study. The after that the wavelet function, the fast Fourier and inverse fast Fourier is used for five band extraction of the signal and the required feature extraction that will later be used to train our machine learning model[66]. We have created a feature pool with the feature we extracted that composed of different kinds of features from the time domain, the frequency domain and the time-frequency domain. Then we recursively picked random feature groups from the androgyny set where as after every iteration the training set was changed to achieve the perfect features. Lastly for the binary classification of cognitive load having high or low values that was related with the physical positioning of the VIP's were determined with the best group of features selected by the feature pool. 


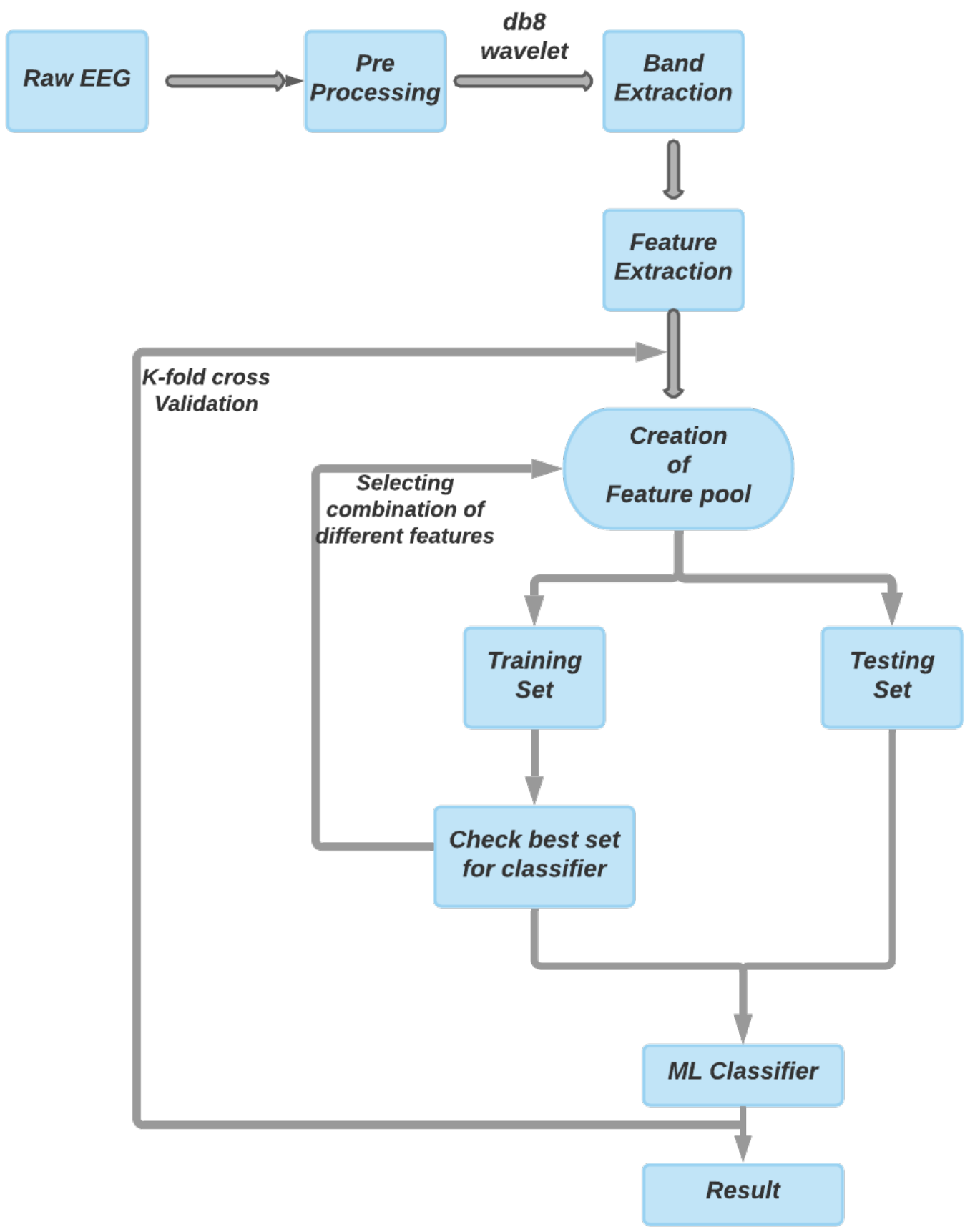

Figure 5.1: System work Flow

\subsection{Pre-Processing of the signals}

When the data was collected the sampling rate was $128 \mathrm{hz}$. There remain a few intramural signal governing system that implys to whatever input an Emotic EPOC+ system obtains. For being analogue as there are lots of conditioning these signals were modified a bit with different cut-off frequencies, like a $0.16 \mathrm{~Hz}$ filter of high pass cut-off, pre-escalated, a $83 \mathrm{~Hz}$ cut-off was used for low pass-filter.Delineation of the filter structure plays a great deal to determine the aggregation of the thinning of each frequency bands.Another sampling at $2048 \mathrm{~Hz}$ was done to exquisite the sig- 
nal a level further.Signals are then notch filtered at 50/60 Hz now that the signals have become digital and lastly down sampling was done to $128 \mathrm{~Hz}$ prior to the main transmission[66]. A notch filter is used to remove a specific interfering frequency.Here it is used as it will create less phase lag in the control loop.

\subsection{Band Extraction}

The Fast Fourier Transform (FFT) was manoeuvred over the EEG data that we got from the headset.Owing to connectivity problems the processed signals were then time domain incorporated for ex positioning missing trials . Then a procedure is applied,a subtraction was done for each of the nine participants along with each of the channels.Brain activity is represented by rhythmic patterns across different frequency bands. Here the db8 wavelet transform was performed to extract five different bands of the EEG signals. They are delta (0.5-4.5)Hz,theta(4.5-7.5) Hz, alpha(7.513.5)Hz,beta(13.5-30.5)Hz,gamma(30.5-60.5)Hz.

\subsection{Feature Extraction}

The surge of a feature is a term that is frequently used to represent a system's practical and non functional qualities..Features can be distinct and unique,EEG signals can be manipulated to produce important feature that maintains a close correlation with the increase and decrease of cognitive load [24]. We extracted different Descriptive Statistical,Pulse and Transition Metrics,Spectral Measurements features for each of the five bands that we got from the wavelet decomposition and for enhancing the quality of each feature we partitioned our each signal into further small groups and later added all the related parts in a single array matrix.We used the matlab toolbox function to extract all the features

The correlation dimension:The uniformly sampled time-domain waveform correlation dimension. We found out that this is a very prominent feature that was never used before in this sector. This calculates the association degree of a screening test time-domain signal of a certain duration in which our sector the segment was 128 data points. The proportionality of the space filled by a group of random points is measured by correlation dimension. The gradient of the correlation integral against the range of resemblance radius is used to calculate it.[68] That has been used as a criterion for distinguishing between unpredictability and external disturbances, as well as for detecting possible flaws.. The corrDim function of the matlab creates a staggered restoration with initially the lag is $T$ and $X 1: X$ with embedding dimension $k$.

$$
\sum_{k=1, k+f}^{T} 1\left(\left\|X_{f}-X_{k}\right\|<L\right)
$$

The indicator function is 1 and radius of similarity is $S$ on which the equation is $S=\operatorname{expo}\left(\right.$ linespace $\left(\log \left(m_{\min }, \log \left(m_{\max }, J\right)\right.\right.$. Now we can see that $s_{\min }$ is the minimum radius, $s_{\max }$ is the maximum radius and $\mathrm{N}$ is the Number of Points. The slope of function defined here is $F(X)$ vs $X$ and the integration of the correlation 
$F(X)$ is defined as

$$
F(N)=\frac{2}{M(M+1)} \sum_{j=1}^{M} M_{j}(R)
$$

Shannon Entropy: Shannon entropy is one of the most important metrics in the areas of information processing. This may be used to determine how much noise is there in a signal.It may be used to relate data about the amount of disturbance, with the greater the level of Shannon entropy, the stronger the amount of entropy, and hence the greater the degree of complexity.It is important to realize the difference between the entropy of a set of promising outcomes, and the entropy of a particular outcome [27].As our signal is non-linear, so this features is very much useful as it evaluates the degree of complexity in a time series. This concept was first discovered by Claude E Shannon. When probability is used to describe the situation, it creates some ambiguity. Shannon entropy includes useful information and alternatives that are unique to transmissions. To sum up,this will overall work as a means of determining order and ambiguity[1].Shannon entropy may be used to determine the extent of turbulence in a central nervous system with computer or machine interface system..e $=$ wentropy $\left(x,^{\prime}\right.$ shannon $\left.^{\prime}\right)$ is the matlab function to compute the shannon entropy where $x$ is the signal and the next parameter is the kind of entropy we want to compute.For a finite discrete random variables $M=m_{1}, m_{2}, m_{3}, \ldots \ldots . m_{f}, m_{j} \in T^{e}$. So the equation is

$$
U(W)=-l \sum q=0^{m} h\left(f_{q}\right) \ln \left(h\left(f_{q}\right)\right)
$$

Here, $l$ is a constant value that serves as an unit of measurement, and $h(f q)$ denotes the probability of $f q$, which is part of the $F$ collection. The equation for $h(f q)$ is:

$$
\sum c=0^{s} p\left(f_{c}\right)=1
$$

Hjorth parameters: The hjorth mobility and complexity is considered as a prominent and robust feature in EEG signals[39].In 1970, Bo Hjorth would be the one to employ it in time-domain signal analysis. The variables are: normalised slope descriptors (NSDs) used in EEG[19]. Three parameters exist in here, Activity, Complexity and mobility. The signal strength energy is represented by the activity parameter, which is the deviance of a function of time. This can be used to represent the frequency response spectrum of a frequency domain. This is described by the equation below:

$$
\text { activity }=\operatorname{variable}(y(x))
$$

where even the signal comes from is represented by $y(t)$ Then comes the hjorth mobility, This measure indicates the energy spectrum's since then or percent of standard deviation. [5]. The square root of the deviation of the first deviation of the transmission $y(x)$ multiplied by the variability of the transmission $y(t)$ defines this.

$$
\text { Mobility }=\sqrt{\frac{\operatorname{variable}\left(\frac{d y(n)}{d n}\right)}{\operatorname{variable}(y(n))}}
$$

Finally, The permanent deformation is represented by the Difficulty parameter. The variable evaluates the transmitter resemblance to a square wave, [5], with a value of 
1 indicating that the signal is more comparable.

$$
\text { complexity }=\sqrt{\frac{\text { Mobility }\left(\frac{d y(n)}{d n}\right)}{\operatorname{Mobility}(y(n))}}
$$

Kurtosis: As said earlier we derived a varieties of feature to explore the signal properly.This is a Higher-Order Statistical feature of the signal.The fourth period (kurtosis) and third period (skewness) of the current waveform reveal insights into dynamic systems in relatively high facts and figures.kurtosis $(\mathrm{X})$ returns the sample kurtosis of the signal X[16].Because our output is a matrix, it produces a covariance matrix containing the sample kurtosis of each transmission column. determines to choose whether or not adjust for bias (flag 0) (flag is 1, the default). When $\mathrm{y}$ indicates a sample from a given population, its kurtosis is biased, meaning means that it differs from the demographic kurtosis by a predictable amount depending on the sample.

$$
X_{k u r t u}=\frac{\frac{1}{L} \sum o=1^{L}\left(y_{o}-\bar{y}\right)^{4}}{\left[\frac{1}{L} \sum o=1^{N}\left(y_{o}-\bar{y}\right)^{2}\right]^{2}}
$$

Crest:The proportion of the biggest absolute value in $\mathrm{x}$ to the rhizosphere (RMS) value of frequency bands is a characteristic. The Matlab function crest $=\operatorname{spectralCrest}(x, f)$ provides a generation of noise signal with arbitrary crest factor of signal $\mathrm{x}$ with frequency $f[61]$.It plots the signal's spectral crest $v$, across time. The form of $g$ determines how the function understands v.

$$
\text { crst }=\frac{\max \left(v_{g \in\left[a_{k}, a_{2}\right]}\right)}{\frac{1}{v_{2}-v_{1}} \sum g=a 1^{n_{2}} e_{k}}
$$

Power Spectral Density:This is a hallmark symptom that has been employed in cognitive load studies in the past. The relative and absolute power in every one of the 14 radio frequencies was first estimated using the Power spectral intensity for each of the 14 channels.Regarding uninterrupted spectra, the power spectral density (PSD) is used. The power utilization of a transmission over a certain frequency range is computed using the integral of the PSD across that frequency spectrum.[46]. The extremes in this continuum, unlike the mean-squared spectrum, do not indicate the power at a specific frequency. The whole power of a signal in the frequency range from DC to halfway of the Nyquist rate is included in a one-sided PSD. The amount of energy in the frequency range from DC to the Nyquist rate is included in a twosided PSD.The matlab function pwelch(x) Welch's overlapping segment aggregating predictor returns the power spectral density (PSD) approximation of the original signal, $\mathrm{x}$. If $\mathrm{x}$ is a vector, it is processed as if it were a single channel. [37]. Every column's PSD is computed separately and placed in the appropriate column of pxx, when the real signal $\mathrm{x}$ is in matrix form. pxx isIf $\mathrm{x}$ is genuine, an exclusive PSD approximation. pxx is a two-sided PSD estimate if $\mathrm{x}$ is complex-valued. By default, $\mathrm{x}$ is separated into the largest portions feasible in order to get as close to, but not more than, 8 sectors with $50 \%$ crossover. A Hamming window is used for each segment[46]. The PSD calculation is computed by averaging the changed invasiveness.. 
Local Maxima:The energy goes up and down with varying maximum amplitude.If $f(x)>=f(x)$ for all $x$ in $X$, a legit function $\mathrm{f}$ given on a scope $\mathrm{X}$ has a global (or absolute) highest rate at $x *$. The matlab function findpeaks(data) outputs a vector containing the input voltage vector's feature points (peaks), datacitemaxima1. A regional maximum is a following assessment that is either greater than or equal to its two nearby values. Non-Inf signal endpoints are excluded. If a top is level, the function just provides the closest indexing value.[30]. In our dataset there were not enough points to meet the required local maxima,so thats why for each of the iteration we had to add some random values to maintain the matrics size and at the end the mean of all the local maxima of a particular given length of signal was passed.

Ratio:Noise and Distortion:This is also another new feature that we used in this sector and it was not previously used before. This is one of the spectrul measurement.The matlab function yields the legitimate sine signal's signal to noise and deformation ratio $(S I N A D)$ in $\mathrm{dBc}$.

$$
R=10 \log _{10}\left(\frac{\sum w=1^{m} x_{w}^{2}}{\sum u=1^{n} x_{u}^{2}}\right)
$$

The (5.10) specifies a seed value for initializing the normal random number generator that is used when adding white Gaussian noise to the input signal.

Singular value decomposition: Another important feature that we extracted was the singular value decomposition of the energy of the signal as a matrix[17]. The Independent Component analysis Breakdown (SVD) of a matrix is a factoring of that matrix into matrices in linear algebra. It possesses some intriguing algebraic features and conveys key geometrical and analytical insights into pseudo signals such as the EEG[32]. It also has some crucial data analytics applications that will aid us in classifying in the future[57]. The overall variation in A must be equivalent to the total of the square of the absolute values. As a result, the size of each unique vector indicates how much of total variation it accounts for.

$$
a_{m n} \approx \sum q=1^{p} u_{m q} s_{q} u_{n q}
$$

wherein $p<n$ denotes the number of absolute values we've chosen to maintain. This will usually be less than the best $10 .(\mathrm{p}=10)(p=10)$ values that are unique. Much of its power comes from its ability to exclude least relevant parts of A via singular value decomposition.

Spurious free dynamic range :The sfdr function in matlab uses a standard periodogram and a Kaiser filter with beta $=38$ to generate the frequency spectrum.Before estimating the power spectrum, we deducted the mean from the signal's specific length. The number of points employed in the discrete Fourier transform (DFT) calculation is proportional to the length of the signal.[43].As a legitimate scalar, the spurious free spatial resolution in $d B$ is defined. The discrepancy in 
$\mathrm{dB}$ between the power at the maximum peak and the strength at the next biggest wavelength is the spurious free dynamic range (spur)[72]. The power estimations are derived by the use of a redesigned periodogram employing a Hamming window because our supply was time series data. When someone wishes to use a different energy spectrum as the foundation for the SFDR calculation, they may use the 'power' flag in the matlab function to enter the power spectrum.[9].

\subsubsection{The ERD/ERS Calculation by a novel approch:}

We calculated the event related a bit differently than the other works, at first we had the block size of 128 as all of the features were calculated to 128 blocks of datapoint within the signal. Then the floor value of the alpha, beta band divided by the block size was taken both for the resting state and the moving state.Then we divided the absolute value of the fast Fourier transform over the time domain with the blocksize. We took the power of the positive half's frequency. At last we calculated the ERDS by taking the ratio of the difference between mean of the resting power and original power with the mean of the resting power multiplied with 100[67].Many studies were done before calculating this with only the Alpha band power[28][4][13], though it is true that Alpha band power plays a huge role in detection of cognitive load, but the Beta and Theta band power also plays similar important for it, so we repeated the process for the Alpha-Beta ratio and Alpha-Theta band power ratio to calculate our cognitive load index which later on acted as the y axis when when fed the data to train and test in our classifier.The ERDS is considered as one of the most prominent and efficient factor to correctly determine the cognitive load of any human being [79] and we modified it a little so that the dispatch and staging gets a higher value.

\subsubsection{Creation of the dynamic feature pool:}

We have chosen some various features from our work of literature review and paper studies. For example: The Hjorth parameter of the bands (Hjorth.m), Shannon entropy of the signal (Shannon.m), Find the local maxima of signal (Pks.m). Aside from that here we have come up with some different features. For instance, If everyone wants to utilize a different power spectral as the basis for the SFDR computation, they could do so by using the 'power' flag in the matworks code editor.singular value decomposition of the signal as a matrix (sVD.m). Initially we selected around ten features for our work. Not necessarily that more features bring more accurate result which is why we used feature elimination technique. For this work we used Python Jupiter Lab. Moreover, we used fourteen dry electrode cells and our sampling rate is $128 \mathrm{~Hz}$. At first,we randomly eliminated one feature and worked on other remaining nine features. Similarly we are recursively eliminating one feature and worked with the rest. Secondly, we randomly eliminated two features and worked on other remaining eight features. Similarly we are recursively eliminating two feature and worked with the rest. We proceed in such a way where we eliminate four features and works with rest of six features. Similarly we are recursively eliminating other four features and worked with the rest. Out of ten features we have selected six features for our works because these features gives more accurate result than other four features. From this we figured out that in future if someone works on same features 
like ours they will get more accurate result if they consider those six features which we selected for our work[25].

\subsubsection{Classification:}

After the creation of dynamic feature pool, many feature vectors were created and were tested in all the available supervised classifiers. All 50 features that were extracted for the five bands along with their initial position and current position were saved in a csv file format.A point here is to be noted that the Hjorth parameters give complex values as a feature which was a bit of a problem as the classifier did not take complex vector as the $\mathrm{x}$ axis to fit into the model. So we came up with a solution with the python sklearn library and we used the label encoder from the pre-processing package so that each row of the column containing the Hjorth values were given a unique value so that they can later be distinguished by any ML classifiers.

We cleaned the csv file before making it fit into any model. We checked for any null values and then we dropped that particular column. The power spectrum feature of the Gamma band was 0 for all of the bands. We dropped that column also.After the dataset was ready to be used we splitted the data and used a 5 fold cross validation with random state equal to 4, That means $80 \%$ of the data was used for training the model and the rest $20 \%$ of the data was used for the test case randomly. Though the Random forest and Gradient Boost algorithm both were at a tie for the performance, finally we chose the Gradient Boost algorithm as our classifier. Gradient boosting performs extraordinarily well as it is a strong out-of-the-box classifier (regressor) that can operate on a dataset with little cleaning work and can adequately explain quasi prior distributions through boosting. All of which clearly matches our feature vectors.Lastly there was creating the $\mathrm{x}$ and $\mathrm{y}$ axis to fit in the model.As we wanted to do binary classification and we had two column as the cognitive load (the Alpha-Beta ratio and the Alpha-Theta ratio), so we created a new column in the csv file with a new algorithm.

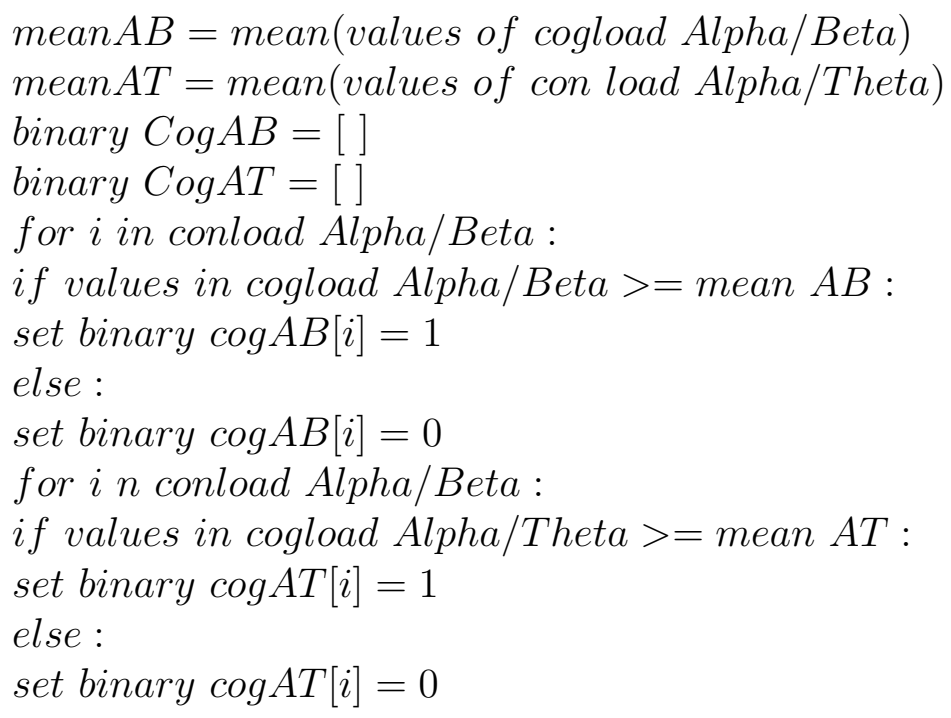

Here 1 means cognitive load high and 0 means low 
By this way a new column was created in our main csv file and that particular column later acted as a the y axis that was fed into the classifier, so our classification was actually binary classification based on whether they have cognitive load at that particular place or not. 


\section{Chapter 6}

\section{Results and Discussion}

This chapter mainly focuses on the various experimental result that were obtained from different experiments with the participants. Many graphs,bar charts and tables are used to help visualize our results for the ease of understanding in different obstacles. Later on the chapter a comparison of our work versus others work has been made to establish superiority of our work.

For performance check we adopted some of the best performance index.Sensitivity,Specificity, Accuracy are some of the traits to measure performance(see eqn $6.1,6.2,6.3$ ). We consider the cases like

- $\mathrm{TP}=$ Cases we correctly predicted to have cognitive load at that place

- $\mathrm{TN}=$ Cases we correctly predicted not to have cognitive load at that place

- $\mathrm{FP}=$ Cases we incorrectly predicted to have cognitive load at that place

- $\mathrm{FN}=$ Cases we incorrectly predicted not to have cognitive load at that place

$$
\begin{gathered}
\text { Sensitivity }=\left(\frac{T P}{T P+F N}\right) 100 \\
\text { Specificity }=\left(\frac{T N}{T N+F P}\right) 100 \\
\text { Accuracy }=\left(\frac{T P+T N}{T P+T N+F P+F N}\right) 100 \\
\text { False pos rate }=\frac{F P}{T N+F P} \\
\text { Time delay }=\left[2 * \frac{T P}{100}+6 * \frac{T N}{100}\right] \\
\text { Quality value }=\frac{10}{\left[\frac{F A}{100}+0.2\right] \times \text { timedelay }} \\
\text { Performance Index }=\frac{T P-F P-F A}{T P} * 100
\end{gathered}
$$




\begin{tabular}{|l||l|l|l|}
\hline \multicolumn{4}{|c|}{ Performance Table 1 } \\
\hline Obstacle & Accuracy & Sensitivity & Specificity \\
\hline Door & $93.23 \%$ & $94 \%$ & $89.67 \%$ \\
Narrow Space & $93.3 \%$ & $94 \%$ & $89.38 \%$ \\
Open Space & $93.42 \%$ & $96 \%$ & $89.67 \%$ \\
Elevator & $93.23 \%$ & $96 \%$ & $89.38 \%$ \\
Stairs & $94.76 \%$ & $96 \%$ & $90.85 \%$ \\
\hline
\end{tabular}

Table 6.1: Performance test(Accuracy,Sensitivity,Specificity) of the GB classifier in different obstacles

After observing all those blind people and measuring all their behaviour by comparatively best classifier method table 6.1 presents that in performance accuracy we get the best result in accuracy $94.76 \%$ at stairs and the performance accuracy is comparatively low at Door and Elevator where both are $93.23 \%$ but in all these five places accuracy is almost close.In case of sensitivity open space,elevator and stairs performance is highest all of them are $96 \%$. and in specificity stairs is the highest $90.85 \%$ and others are close to $89.5 \%$.In table 6.1 we get one thing is common that in case of accuracy sensitivity and specificity at Stairs the rate is high and it gives better performance.

\begin{tabular}{|l||l|l|l|l|l|}
\hline \multicolumn{7}{|c|}{ Performance Table 2 } \\
\hline Obstacle & $\begin{array}{l}\text { Null Ac- } \\
\text { curacy }\end{array}$ & $\begin{array}{l}\text { False } \\
\text { Alarm }\end{array}$ & $\begin{array}{l}\text { Time De- } \\
\text { lay }\end{array}$ & $\begin{array}{l}\text { Quality } \\
\text { Value }\end{array}$ & $\begin{array}{l}\text { Performance } \\
\text { index }\end{array}$ \\
\hline Door & $78.75 \%$ & $10.3 \%$ & $74.33 \mathrm{~ms}$ & $41.94 \%$ & 2086.23 \\
Narrow Space & $10.61 \%$ & $94 \%$ & $74.421 \mathrm{~ms}$ & $41.87 \%$ & 2082.94 \\
Open Space & $10.3 \%$ & $94 \%$ & $74.38 \mathrm{~ms}$ & $41.98 \%$ & 2088.22 \\
Elevator & $10.61 \%$ & $94 \%$ & $74.42 \mathrm{~ms}$ & $41.87 \%$ & 2082.94 \\
Stairs & $9.08 \%$ & $94 \%$ & $74.02 \mathrm{~ms}$ & $42.2 \%$ & 2100 \\
\hline
\end{tabular}

Table 6.2: Performance test(Null Accuracy,False Alarm,Time Delay,Quality Value) of the GB classifier in different obstacles

Table 6.2 shows that the best result for null accuracy is observed against the door which is $78.75 \%$. The other results of null accuracy are comparatively lower and closer to one another. This is the reason that the false alarm rate is the lowest at the door which is $10.3 \%$ and the other values are much more which is above $90 \%$. However, the quality value for the door, narrow space, open space and elevator are the same but it is a bit higher on the stairs.

Basically, in gradient boost using estimators parameter which initial predictions are computed using an estimator object. The number of boosting stages that must be completed. Gradient boosting is somewhat impervious to over fitting, but a greater number normally yields stronger outcomes. Generally, the default estimator is 100 . In table 6.3 we are starting estimators by 100 which gives similar accuracy in door and narrow spaces. We recorded the highest accuracy on the stairs. After that, we perform 130 estimators which boost the performance accuracy for all places except stairs. Here stairs provide the same accuracy like before. Next, we increase estimators to 150 which again increase accuracy of all positions. Moreover, we got higher accuracy from open space here. For 200 estimators we got almost the same 


\begin{tabular}{|l||l|l|l|l|l|}
\hline \multicolumn{7}{|c|}{ Obstacles } \\
\hline estimators & Door & Narrow & Open & Elevator & Stairs \\
\hline 100 & $92.17 \%$ & $92.17 \%$ & $92.20 \%$ & $92.11 \%$ & $92.23 \%$ \\
130 & $92.29 \%$ & $92.36 \%$ & $92.79 \%$ & $92.29 \%$ & $92.23 \%$ \\
150 & $92.61 \%$ & $92.48 \%$ & $93.73 \%$ & $92.61 \%$ & $92.54 \%$ \\
200 & $92.95 \%$ & $92.98 \%$ & $92.87 \%$ & $93.05 \%$ & $92.67 \%$ \\
250 & $93.15 \%$ & $93.05 \%$ & $92.67 \%$ & $93.05 \%$ & $92.79 \%$ \\
280 & $92.92 \%$ & $92.92 \%$ & $92.79 \%$ & $93.05 \%$ & $92.67 \%$ \\
300 & $92.79 \%$ & $92.79 \%$ & $92.67 \%$ & $93.05 \%$ & $92.79 \%$ \\
\hline
\end{tabular}

Table 6.3: Changes in the performance of the GB as we iterate over the number of estimators

accuracy from doors and narrow spaces. In 250 estimators door space gave best accuracy among all. After that, from 280 estimators the doors accuracy fell down but elevator space gave higher performance. Lastly, when we use 300 estimators elevator space again holds higher accuracy.

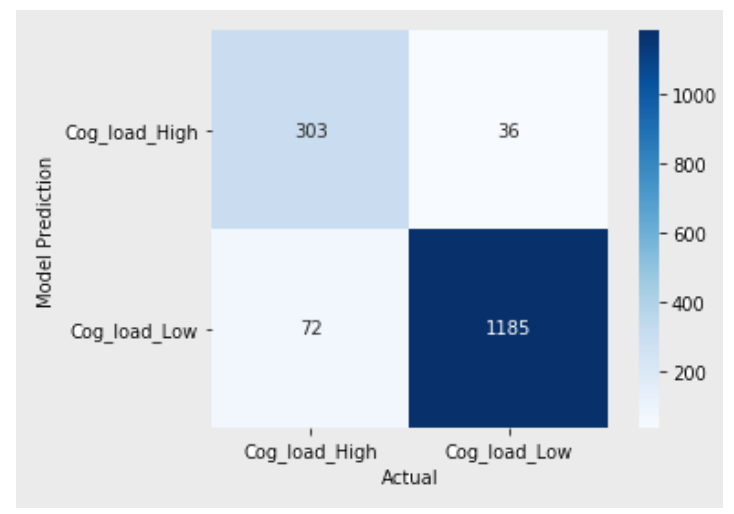

Figure 6.1: confusion matrix at door

A confusion matrix is used to measure the efficiency of a classification algorithm. Using only classification accuracy may not give the best results if the number of observations is unequal in each class. To know if the classification model is getting right or not, confusion matrix can be a good option.

In Fig.6.1 ) the false negative and false positive value is comparatively lower which is a good sign because those values are considered to be predicted values. Whereas the actual value which is true positive and true negative are on the greater side. Here, the true positive value for the door is the highest so we can come to a point that there is a good prediction for door and it will give a precise signal to the blind man. This information can only be known from the confusion matrix.

In Fig.6.2, we see the same thing as Fig.6.1 As the true positive value is the highest in actual value so there will be also be correct prediction on elevator. Also, the value of false negative for both of the figures are the same which is 72 .

If we see Fig.Fig.6.3, here the true negative value is the highest. So we can correctly predict that the blind man does not have cognitive load at that place means he was not at that place. For Fig.6.4,here the true positive value is the highest. So, we can correctly predict that the blind man has cognitive load at that place. Wrong prediction amount is comparatively less at open space. Fig.6.1 and Fig.6.2.TN combined 


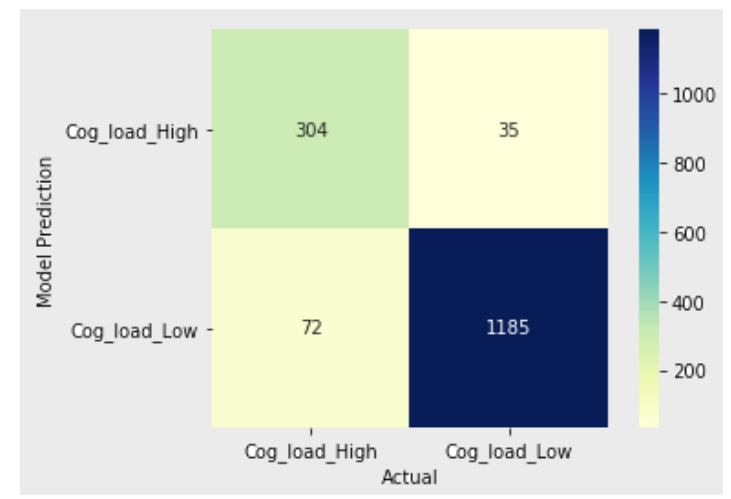

Figure 6.2: confusion matrix at elevator

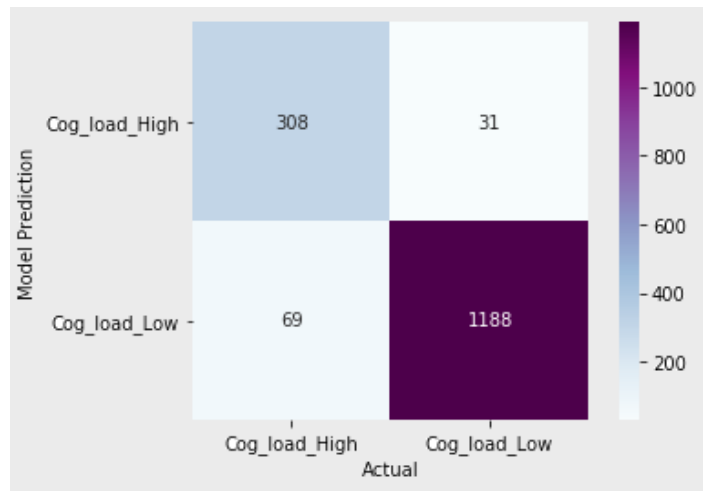

Figure 6.3: confusion matrix at narrow

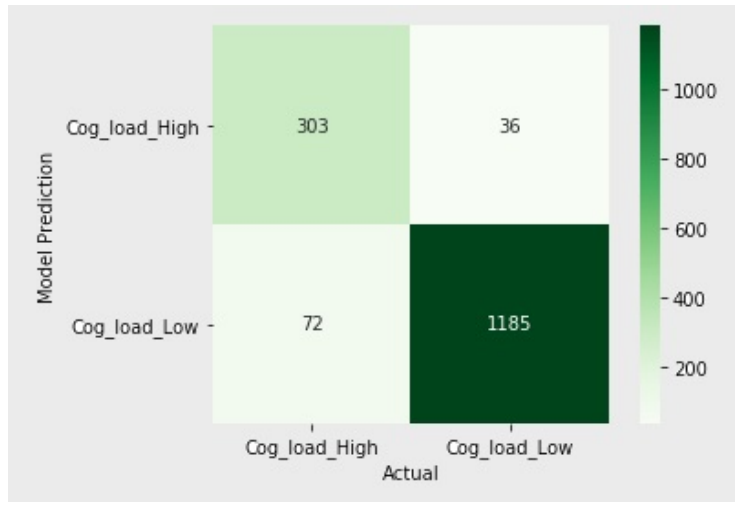

Figure 6.4: confusion matrix at open

is almost $94 \%$ which gives us the information that our classifier accuracy is high and the false prediction is low.

Confusion Matrix is a performance measurement for a machine learning classification problem where output can be two or more classes. When the number of findings in each class differs, depending entirely on classification accuracy would not provide the best results. To know if the classification model is getting right or not, a confusion matrix can be a good option. Here In this figure 6.5 confusion matrix we can see that out of 1596 cases 307 cases are True Positive 1187 people are True Negative, 70 cases are False Negative and 32 are False Positive. The true values determine whether the person has cognitive load at stairs or not. Our primary goal is to keep 


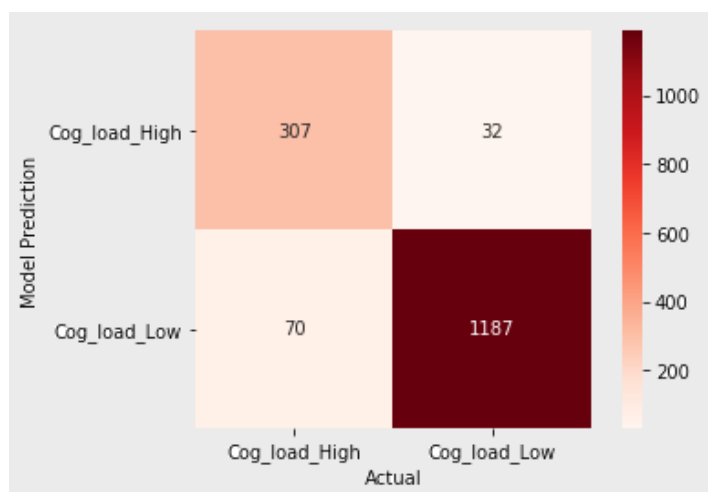

Figure 6.5: confusion matrix at stairs

TP and TN as high as possible and here the addition of TP and TN combined is almost $94 \%$ which gives us the information that our classifier accuracy is high and the false prediction is low.

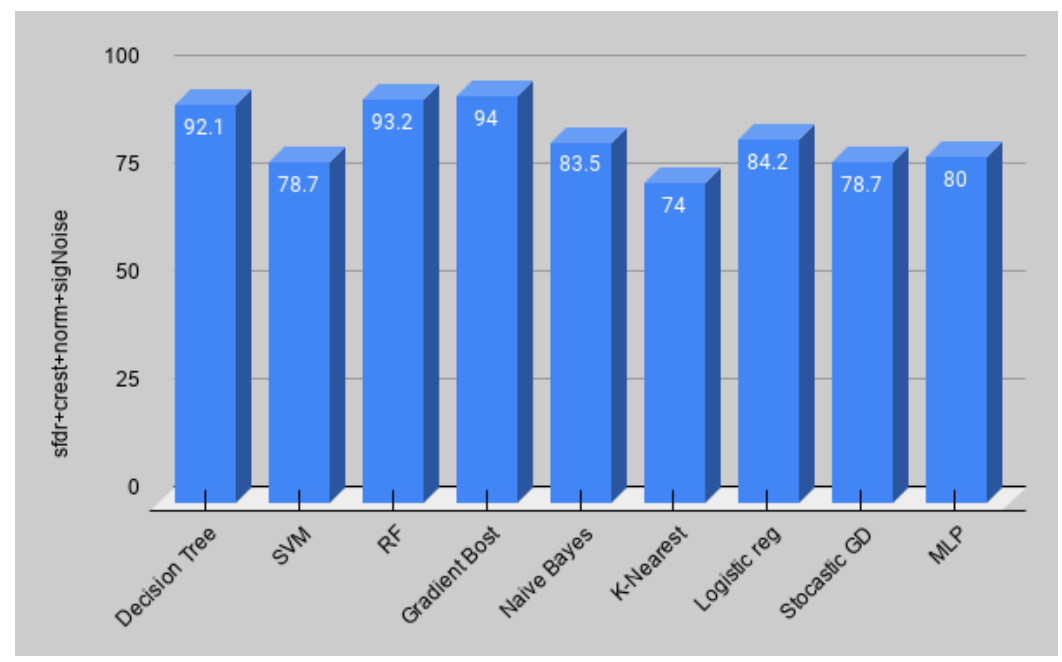

Figure 6.6: Main Feature at AB

In this Figure 6.6 After processing the EEG signal we got 9 features our goal was to find the best classifier after including and excluding different sets of features .we have measured it by focusing mainly on the accuracy rate on event-related desynchronization/synchronization (ERD/ERS).In this Figure 6.6 after excluding Sfdr ,crest,norm and sigNoise and focusing on ALPHA BETA band power performance the performance of the gradient boost is high it beats random forest and decision tree here where decision tree accuracy is 92.1 and the random forest is 93.2 here all the other classifier performance is below margin but gradient boost is above all.

In figure 6.7 after excluding sfdr ,crest,norm and sigNoise and focusing on ALPHA THETA band power performance the performance of the gradient boost is high it .the accuracy rate is almost 94 percent where the decision tree is 91.9 and the random forest is 92.3 and the others are below average .in most of the cases people measure classifier accuracy based on ALPHA BETA band power but here ALPHA THETA BAND measurement adds a new era which will be helpful for the other researchers

In most of the research papers they have worked with alpha band power but in our work we have tried to imply different ratios of power bands like alpha-beta and 


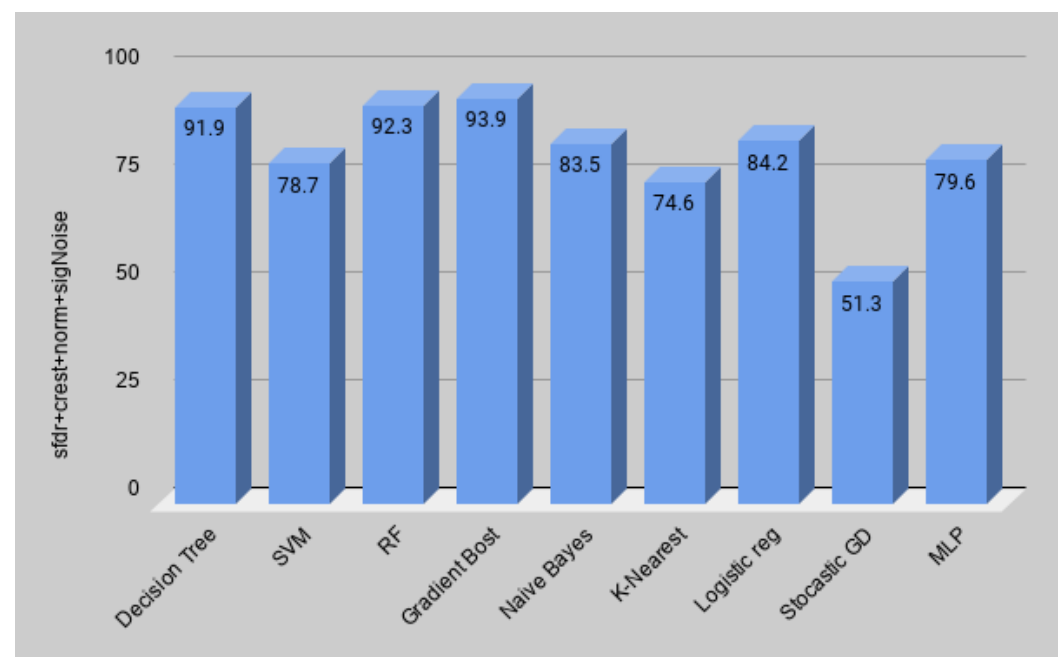

Figure 6.7: Main Feature at AT

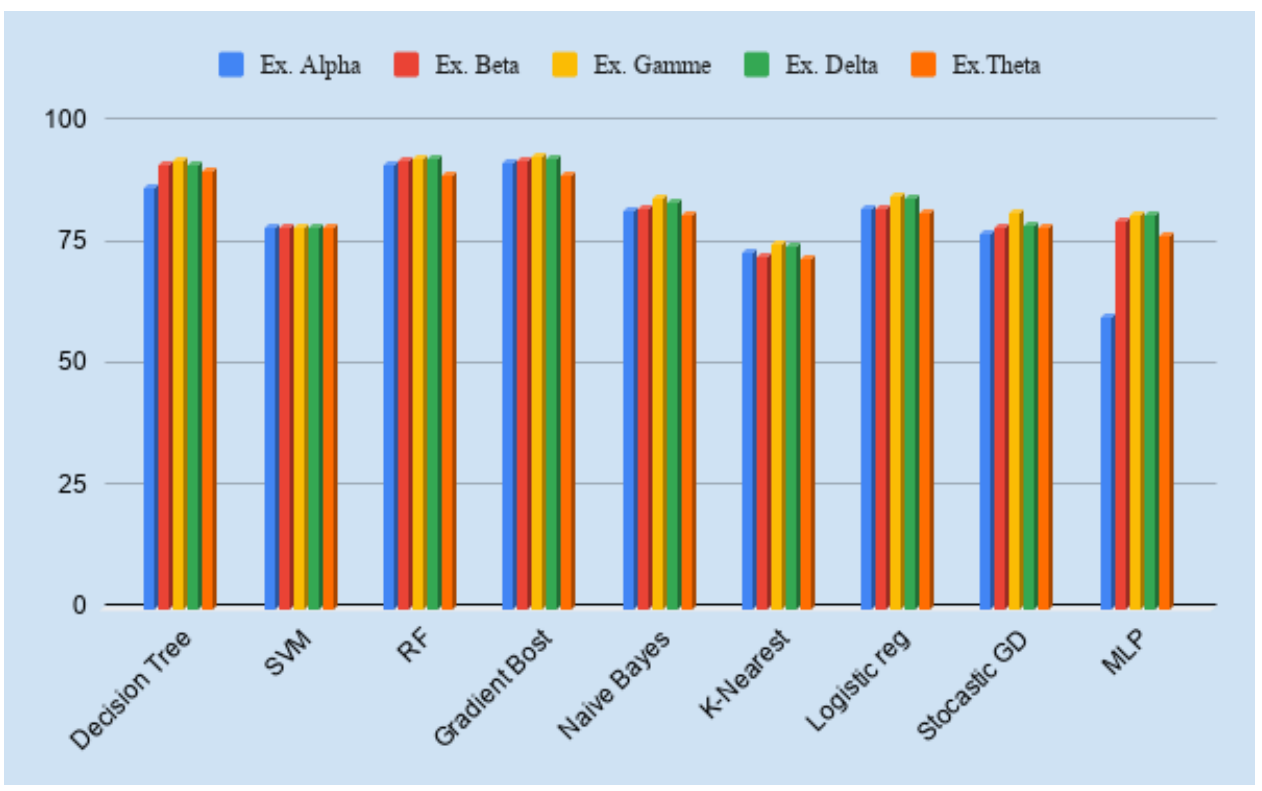

Figure 6.8: classifier selection from alpha-beta bands ratio

alpha-theta. In the alpha-beta ratio power band, firstly we excluded alpha and measured accuracy with other bands in our classifier.Similarly, we excluded other bands recursively and works on rest of the bands. in each turns and testing accuracy we have come to conclusion that we get best result with Gradient boost and random forest classifier which is show in 6.8 But if we closely observe in 6.8 we can see that in gradient boost when we exclude gamma bands we get more accuracy with others. Additionally, when we work with alpha-theta ratio band power again similarly we exclude one band after another and work with the rest of them for finding the best accuracy. Here in 6.9 we have mixed experience that in the decision tree we get good results when our gamma band was excluded. On the contrary, SVM, K-rearest and NLP did not give higher accuracy. However, we get the best result from gradient boost again. In 6.9 We can see when we excluded our gamma band the rest of the other bands gave higher accuracy in our work. From dynamic feature pool the features we have chosen are selected through the process which is the ratio of band 


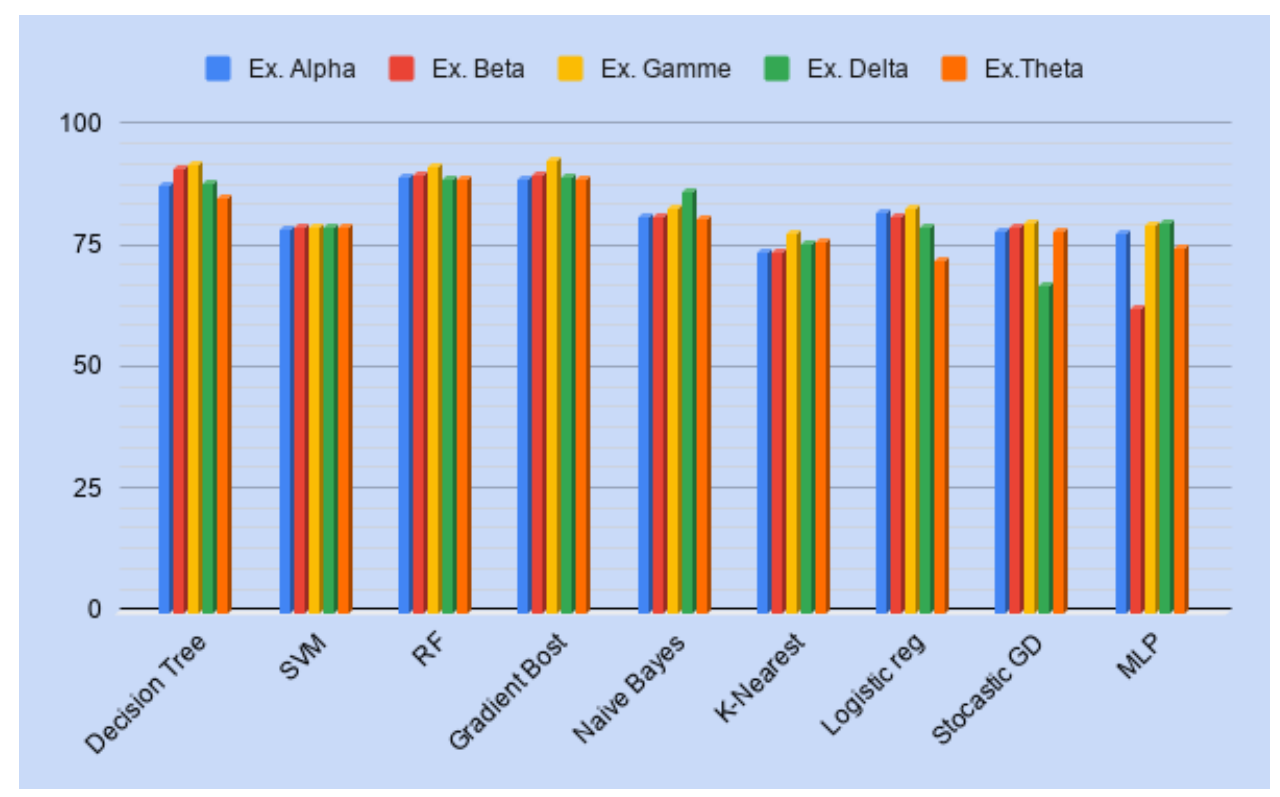

Figure 6.9: classifier selection from alpha-theta bands ratio

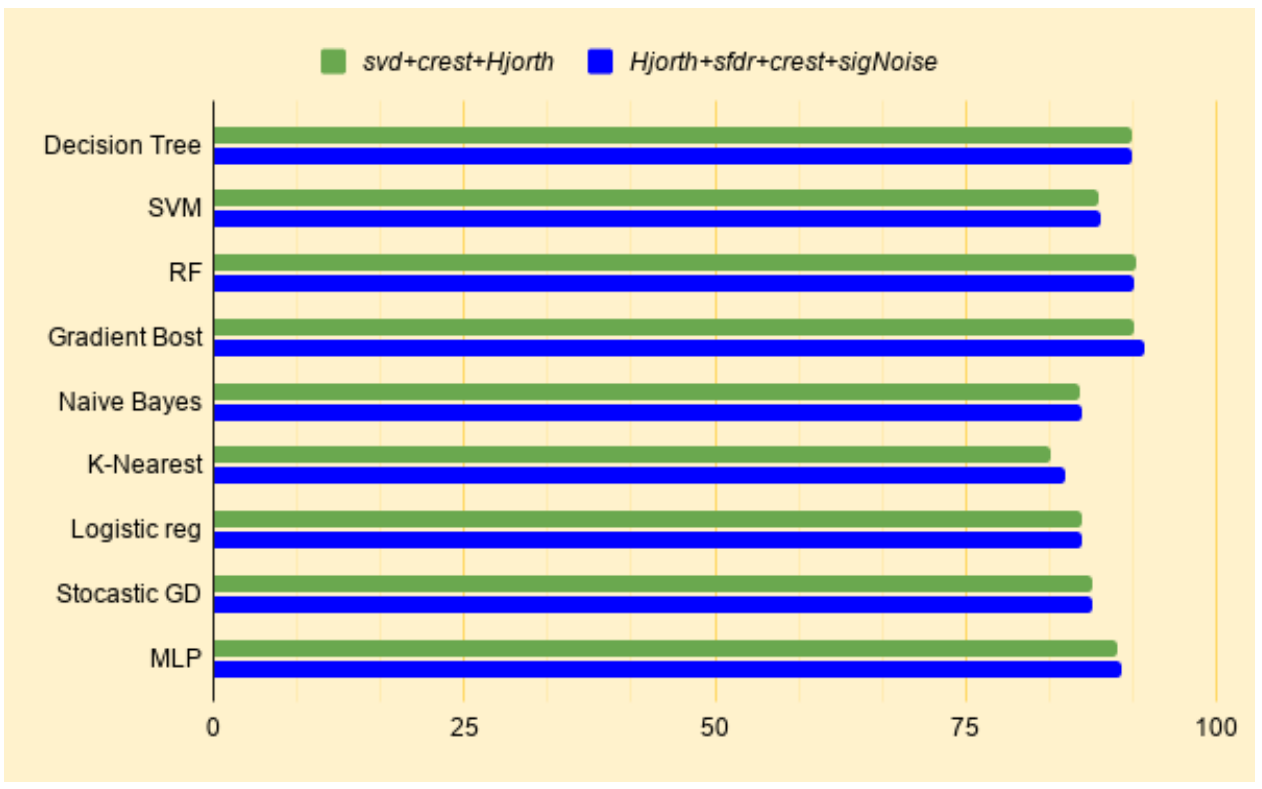

Figure 6.10: features selection in alpha-beta band ratio

power for example: alpha-beta and alpha-theta. In alpha-beta band power ratio firstly, we exclude svd, crest, hjorth and using the rest of the features in classifier which provide us the result of green-line in 6.10. After that we exclude hjorth, crest, signoise, sfdr and using the rest in classifier we get the result for blueline. So we can come to this conclusion after observing this result graph that gradient boost classifier gives more accurate result when we exclude hjorth, crest, signoise and sfdr among all classifiers.

Afterwards, when we work with alpha-theta ratio band power firstly we used seven features in classifier and rest of the three features which are svd, crest and hjorth are excluded to sum up the result that is shown in green-line of 6.11 . Consequently, we exclude these four features hjorth, crest, signoise and sfdr and worked on rest of the features in classifier to land on the green-line result in 6.11. Analysing the 


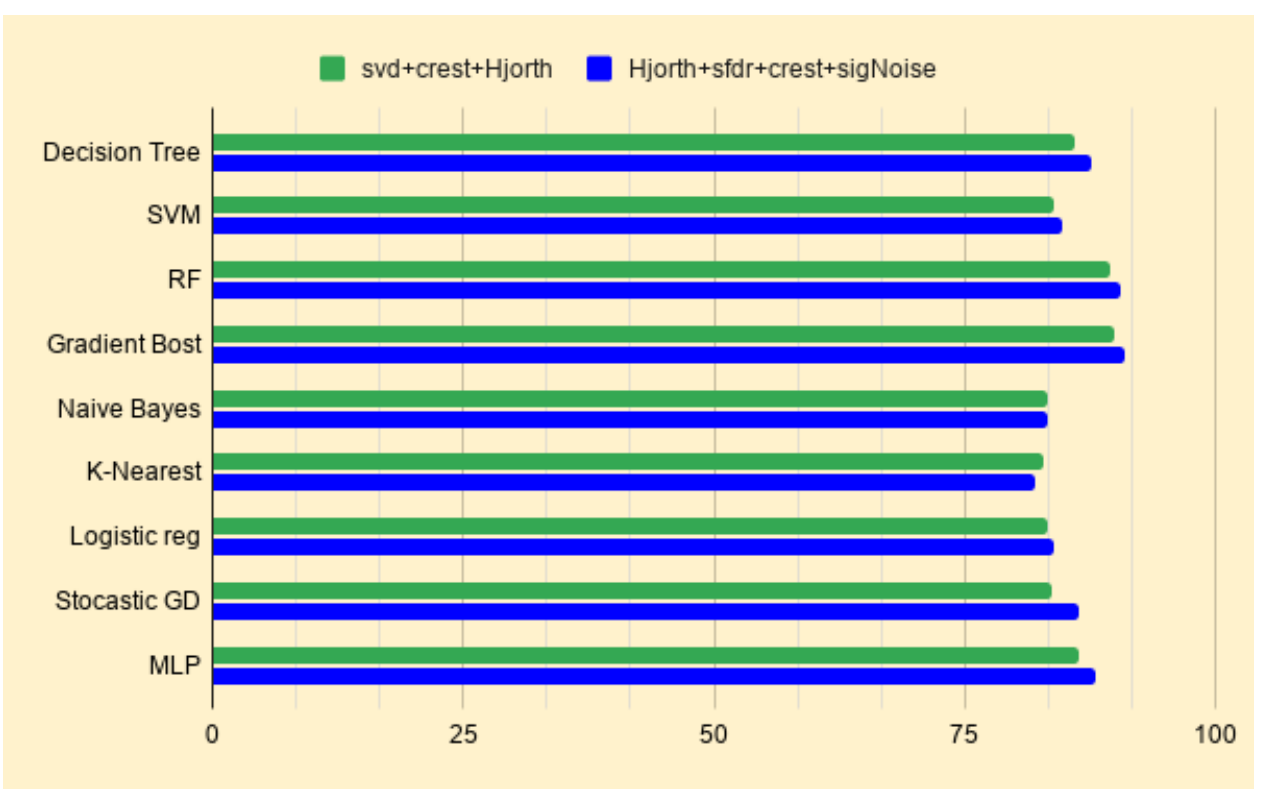

Figure 6.11: features selection in alpha-theta band ratio

result we see that again gradient boost classifier gives a higher accuracy than other classifiers in case when we eliminate those four features hjorth, crest, signoise and sfdr.

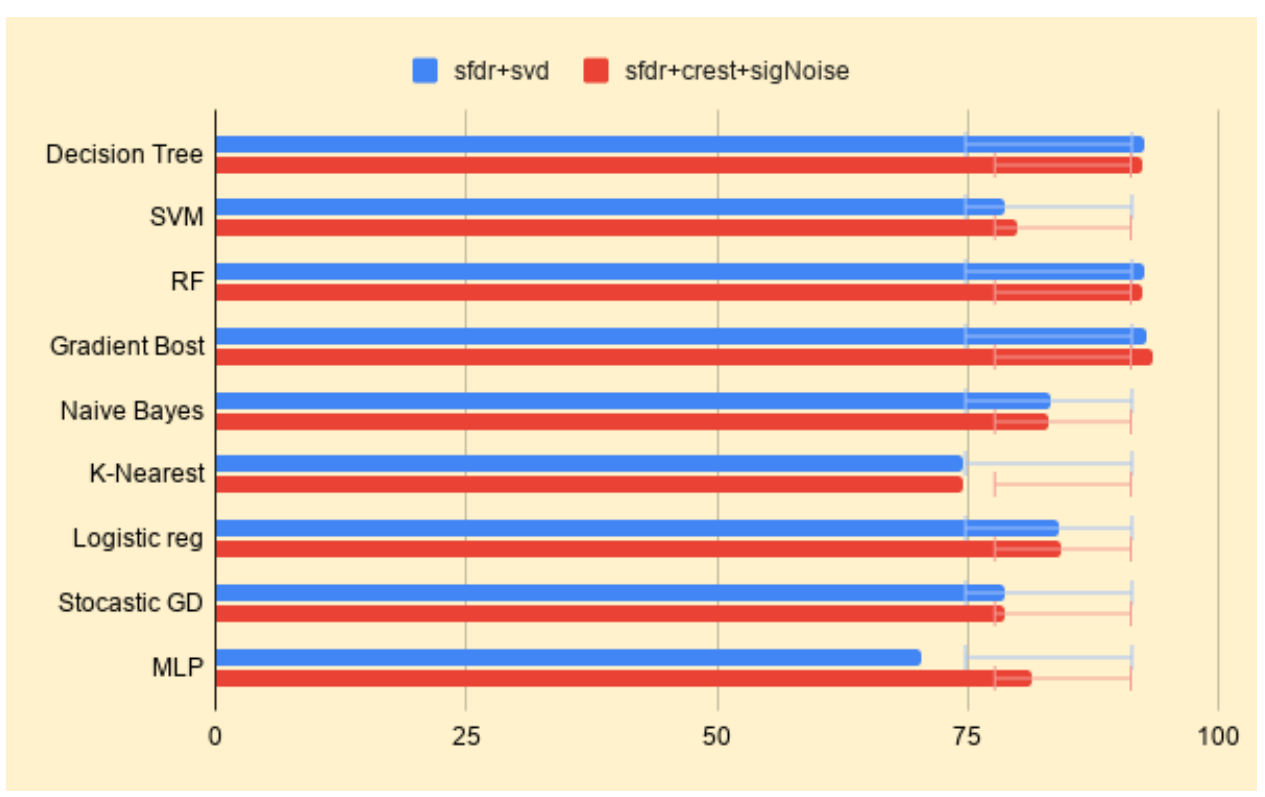

Figure 6.12: ROC curve for the Alpha-Beta ratio

6.12 and 6.13 is the result of keeping the Hjorth parameters in the classifier along with different combination of features, the blue horizontal bar represents the accuracy of all the classifiers when singular value decomposition the power spectrum calculated using a kaiser window is excluded from the classification and the red bar indicates crest factor, signal noise to distortion ratio and the power spectrum exclusion accuracy of different supervised classifiers. The $\mathrm{X}$ asis is the accuracy percentage out of 100 and the $\mathrm{Y}$ axis is the name of all the classifiers. Actually, we did this kind 


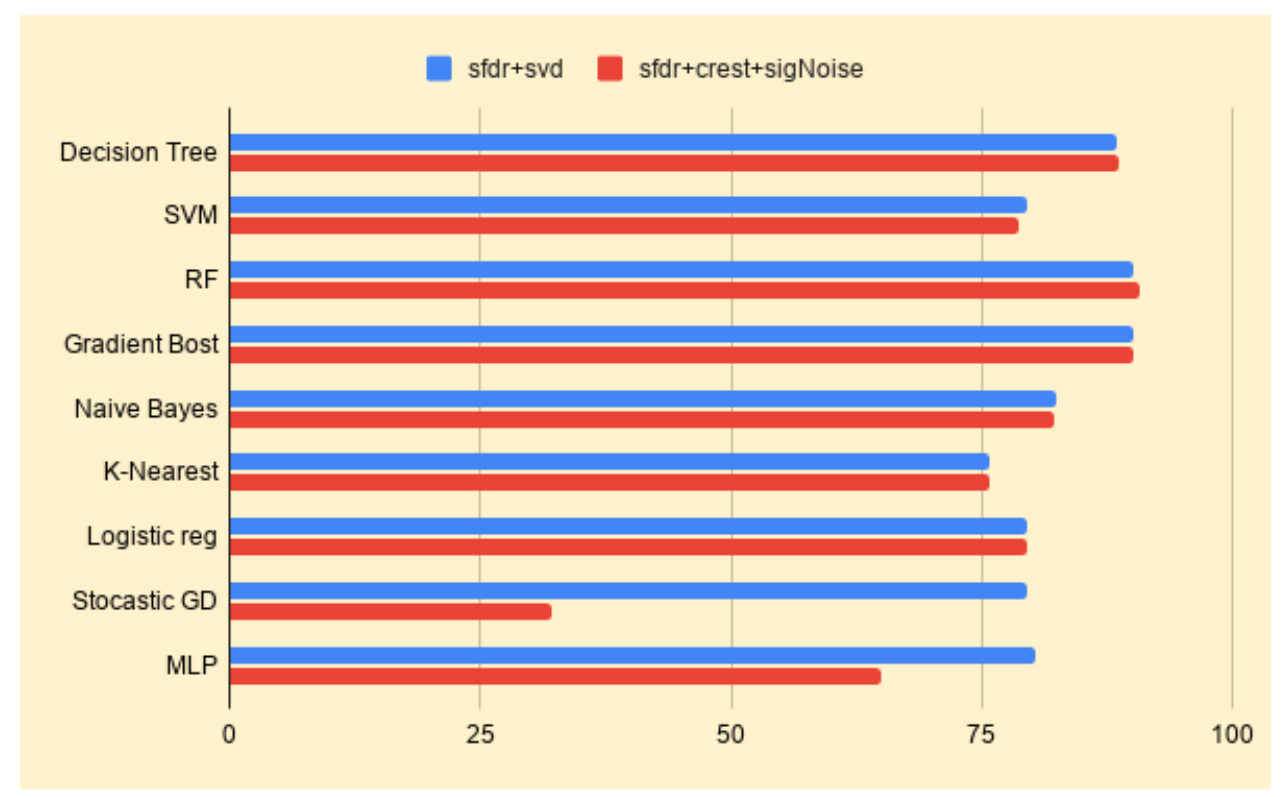

Figure 6.13: ROC curve for the Alpha-Beta ratio

of classification to check if there was any kind of special factor that may affect the classifiers to perform differently, which was proved to be true if we closely observe the Fig 6.10 and Fig6.11.

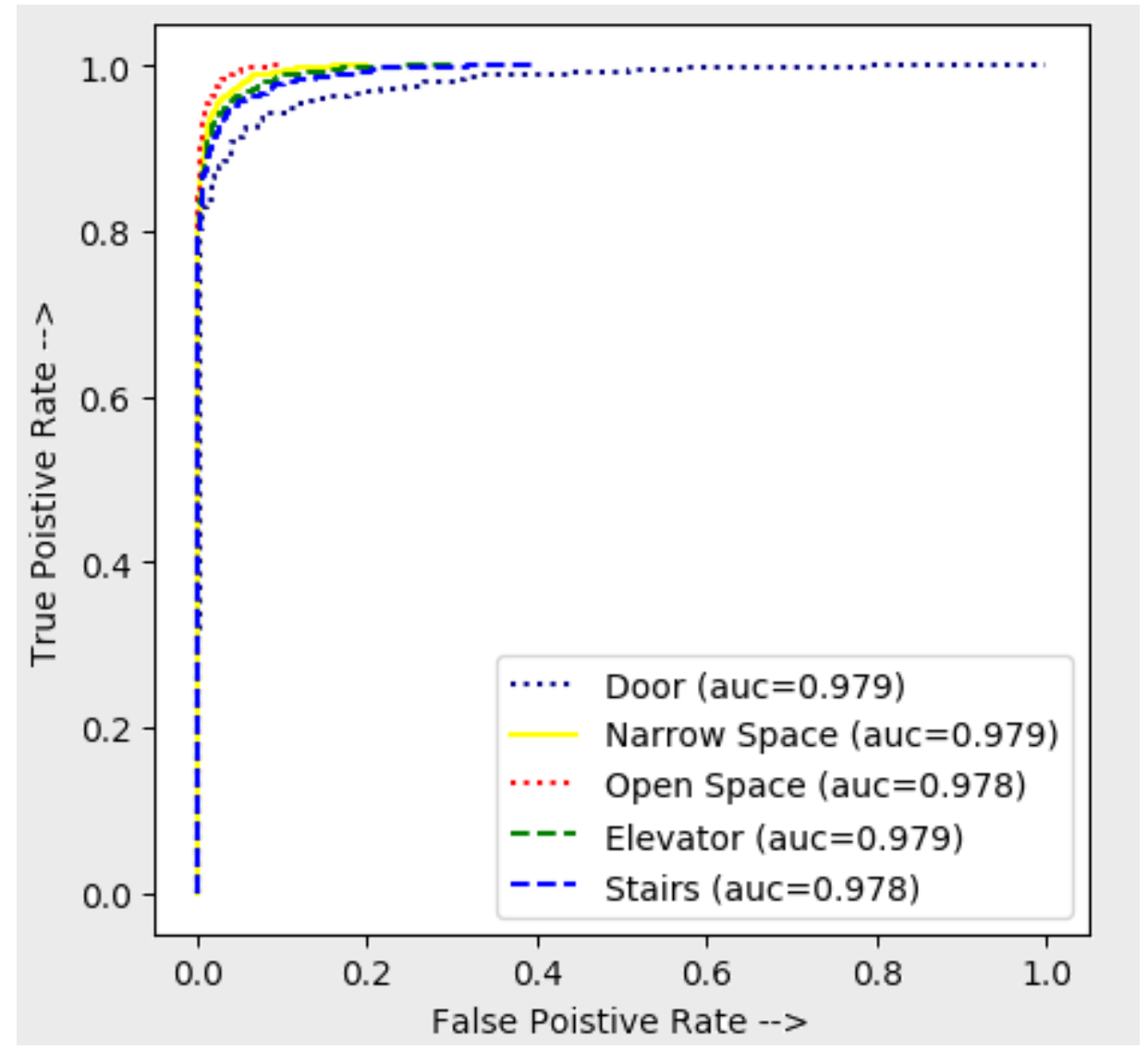

Figure 6.14: ROC curve for the Alpha-Beta ratio

In both alpha-beta and alpha-theta ratios we came to this decision that when we 
exclude those four particular features hjorth, crest, signoise and sfdr in our classifier. In both of the graphs we can see that gradient boost classifier provides best accuracy than others. So, that is why we chose those six particular features for our work.

For a binary classification like ours The Receiver Operator Characteristic (ROC) curve works as the evaluation metric. This curve represents the probability that plots the True positive rate that we got from the confusion matrix against False positive rate at varied entry levels and predominantly distinguishes the 'signal' making it apart from the 'noise'. The Area Under the Curve is the indicator of a classifier's capability to differentiate among classes. As seen in fig 6.14 Open space achieved the highest position in the axis for the Alpha-Beta ratio band power, followed by the narrow space.It is said that the higher the AUC and the ROC, the more preferable the effectiveness of the pattern at differentiating within negative and positive class. Thirdly the elevator comes in position, Though all of their AUC is almost same, we did not use weighted AUC in the visualization as it has nothing to do with the class balancing but only interested in certain regions of the ROC sace with higher recall.Door gives the worst graph performance as shown in the figure 6.14

The figure 6.15 represents the ROC curve of the five obstacles for the Alpha-Theta

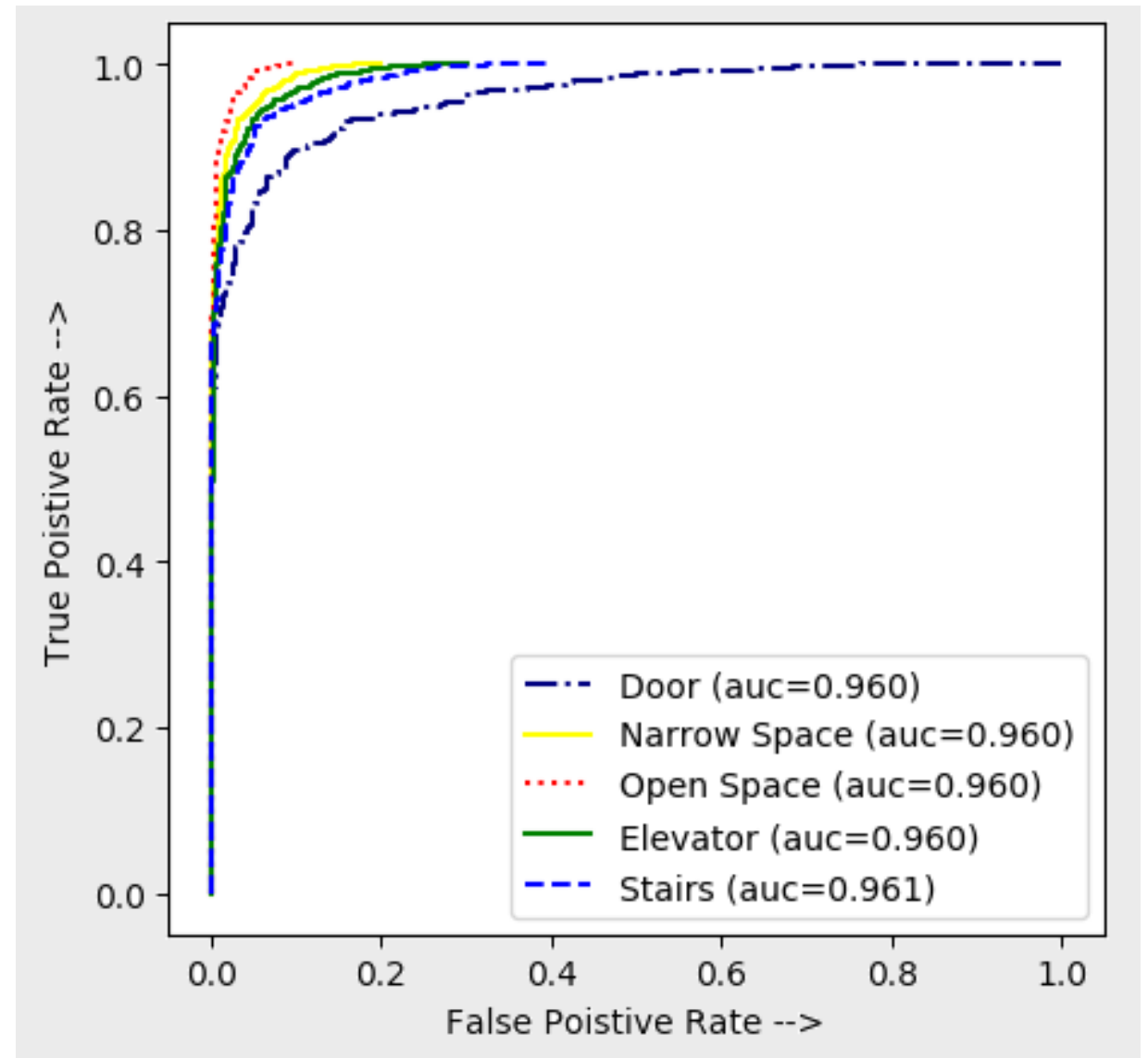

Figure 6.15: ROC curve for the Alpha-Theta ratio

ratio.Again like the previous figure 6.14 the Open space has the highest Roc curve followed by the narrow space, but we can see the change in the curve of the stairs, as it falls down a bit compared with the Alpha-Beta band power. This is actually the reason we decided to use ratio of the band powers, to see how much impact they imply on the change of the results.However our least accuracy is over the $90 \%$ which 
easily indicates the solidity and the novel work that we did by creating a recursive feature pool and taking the Ratio of the band powers (not only the Alpha band power like the previous works) is better than previous methods.

\subsection{A Comparative study:}

Through meticulous and scrupulous detailing we have come to decide that our work was better than any of the previous work done in the same field.

- Zubayed et al. used all entropy based features in their work [64] and got better accuracy than us only in narrow space and door which was $96 \%$ and $94 \%$, but as our work was conducted on various other signal based features, we acquired better accuracy in other indoor obstacles, this also is applicable for specificity and sensitivity

- Rayhan et al.[70] considered only the Gamma,Theta and Beta band for their work for ERDS comparison where the Alpha band is a must for this index calculation, they gained an accuracy below $90 \%$ for all obstacles but as we have used the Alpha band in the CLI calculation, all of our accuracy was above $92 \%$.

- Luo et al. used support vector machine (SVM) to learn their classifier for the (Motor Imaginary) MI tasks. Moreover, they ware used the 10-fold crossvalidation for their work[82]. However, this makes overlapping of data more frequent than needed and as a result their accuracy was below $90 \%$.

- Charalampos Saitis et al. opted to concentrate on the alpha bands since brain activity at those frequencies has been linked to cognitive strain in a number of studies of task. On the contrary we chose alpha beta and alpha theta band ratios resulting in a better performance index. accuracy[63].

- Kyriaki Kalimeri et al. used the commonly used learning method Random Forest for classification since it can handle potentially associated predictor variables and gives a simple assessment of variable relevance. Whereas, we decided to go with Ensemble type Gradient Boost classifier[47]. We could easily manipulate the performance through manipulation of the number of estimators, where RF manipulation with the number of trees in the forest was hard for a non-linear signal like EEG.

- R. Panda et al.[27] used seizure signal from hippocampal region which is the inner (medial) area of the temporal lobe for analysis while we analyzed the frontal area of the brain in our paper which could create a more clear visualization and realization of the cognitive load of the brain.

\subsection{Future Works:}

In our research we ware taking already prepossessed data that is why we have limited scope for some innovative works. We are hopeful that in future we will work on raw signal data which give us high Chances to do something new. Moreover we aware 
that, we have data those are tested on limited number of people which are not represent the mass population. We need more variety of people which might be give us several types of raw signal which give more accurate result for real world implementation. Furthermore, we work on indoor mobility with some circumstances. However, we hopeful that we will increase the number of obstacles in future which produce high chance to give us better real time solution. In our work we are working on binary class classification system which not represent unsupervised learning. In future we will work on multi class classification which gives us scope to work on unsupervised learning algorithm in machine learning. In fact that, In future we are committed to Increase our research area for instance, We will work on outdoor mobility also, even so, we will not only bounded to visual impaired people but also works with deaf and ear less people.

\subsection{Conclusion:}

Habitually alluded as AMD, age-related muscular degeneration is one the leading cause of visual impairment and close-up vision among people. This results in damage to one's central vision, navigation and other day to day life work. The framework that we presented here was a development of the previously studies in the related field. Based on our approach, we predicted the cognitive load of different degree of sight loss among VIP's and their current position based on the analysis of EEG signals with the supervised machine learning algorithms. As we previously labelled our data with the ERDS marker of cognitive load, supervised learning was a great success in our prediction. We upgraded the index by combining the baseline band power of the Alpha-Theta ratio and Alpha-Beta ratio because the Theta and Beta bands also play very important role for detecting changes in cognitive load of the brain. As we mainly worked with the working memory of the brain, we created a hybrid feature pool and made sets of test features by combining features from the Descriptive Statistics,Pulse and Transition Metrics and Spectral Measurements features and recursively tested and trained our model with all the supervised Machine Learning classifiers. Among them, two famous ensemble classifiers, The Random forest and Gradient Boost classifier has given the best performance. We finally chose the Gradient boost classifier which gave $93.23 \%$ accuracy for door, $93.3 \%$ for Narrow Space, $93.42 \%$ for Open Space, $93.23 \%$ for elevator and finally for stairs it gave $94.76 \%$ accuracy for correctly predicting the cognitive load.

A special discovery happened during our thesis. The hjorth parameters when used as feature to train machine learning models all the supervised classifiers except the Random Forest,Decision tree and Gradient Boost, the accuracy of the classifiers falls severely. The SVM classifier, if used for detection of cognitive load, must be trained without the hjorth parameters because the accuracy fall almost by $20-26 \%$ for this. Another thing to notice here is that when the ratio of band power of the signal bands are used together rather than using only the Alpha band, the accuracy gets a boost up.Our work has paved a clear path for designing a smart app based or monitoring system of guiding VIP's along the unfamiliar indoor mobility and will be uncomplicated the total scenario for them to overcome obstacles. 


\section{Bibliography}

[1] C. E. Shannon, "A mathematical theory of communication", The Bell System Technical Journal, vol. 27, no. 3, pp. 379-423, 1948. DOI: $10.1002 /$ j.15387305.1948.tb01338.x.

[2] R. Hari, N. Forss, S. Avikainen, E. Kirveskari, S. Salenius, and G. Rizzolatti, "Activation of human primary motor cortex during action observation: A neuromagnetic study", Proceedings of the National Academy of Sciences, vol. 95, no. 25, pp. $15061-15065,1998$.

[3] W. Klimesch, "Eeg alpha and theta oscillations reflect cognitive and memory performance: A review and analysis", Brain research reviews, vol. 29, no. 2-3, pp. 169-195, 1999.

[4] C. M. Krause, L. Sillanmäki, M. Koivisto, C. Saarela, A. Häggqvist, M. Laine, and H. Hämäläinen, "The effects of memory load on event-related eeg desynchronization and synchronization", Clinical neurophysiology : official journal of the International Federation of Clinical Neurophysiology, vol. 111, pp. 20718, Dec. 2000. DOI: 10.1016/S1388-2457(00)00429-6.

[5] M. Vourkas, S. Micheloyannis, and G. Papadourakis, "Use of ann and hjorth parameters in mental-task discrimination", in 2000 First International Conference Advances in Medical Signal and Information Processing (IEE Conf. Publ. No. 476), 2000, pp. 327-332. DOI: 10.1049/cp:20000356.

[6] H. Adeli, Z. Zhou, and N. Dadmehr, "Analysis of eeg records in an epileptic patient using wavelet transform", Journal of neuroscience methods, vol. 123, no. 1, pp. 69-87, 2003.

[7] F. Paas, J. E. Tuovinen, H. Tabbers, and P. W. M. V. Gerven, "Cognitive load measurement as a means to advance cognitive load theory", Educational Psychologist, vol. 38, no. 1, pp. 63-71, 2003. DOI: 10.1207/S15326985EP3801 \ _8. eprint: https://doi.org/10.1207/S15326985EP3801_8. [Online]. Available: https://doi.org/10.1207/S15326985EP3801_8.

[8] R. Parasuraman, "Neuroergonomics: Research and practice", Theoretical Issues in Ergonomics Science, vol. 4, no. 1-2, pp. 5-20, 2003. DOI: 10.1080/ 14639220210199753. eprint: https : / doi.org / 10.1080/ 14639220210199753. [Online]. Available: https://doi.org/10.1080/14639220210199753.

[9] N. Ghittori, A. Vigna, and P. Malcovati, "Analysis of the ideal sfdr limit for an n bit digital-to-analog converter", in 2005 12th IEEE International Conference on Electronics, Circuits and Systems, 2005, pp. 1-4. DOI: 10.1109/ICECS.2005. 4633448 . 
[10] A. Subasi and E. Ercelebi, "Classification of eeg signals using neural network and logistic regression", Computer methods and programs in biomedicine, vol. 78, no. 2, pp. 87-99, 2005.

[11] J. Żygierewicz, P. Durka, H. Klekowicz, P. Franaszczuk, and N. Crone, "Computationally efficient approaches to calculating significant erd/ers changes in the time-frequency plane", Journal of Neuroscience Methods, vol. 145, no. 1-2, pp. 267-276, 2005.

[12] R. L. Klatzky, J. R. Marston, N. A. Giudice, R. G. Golledge, and J. M. Loomis, "Cognitive load of navigating without vision when guided by virtual sound versus spatial language.", Journal of experimental psychology: Applied, vol. 12, no. 4, p. 223, 2006.

[13] P. Missonnier, M.-P. Deiber, G. Gold, P. Millet, M. Gex-Fabry, L. Fazio-Costa, P. Giannakopoulos, and V. Ibáñez, "Frontal theta event-related synchronization: Comparison of directed attention and working memory load effects", Journal of neural transmission (Vienna, Austria : 1996), vol. 113, pp. 147786, Nov. 2006. DOI: 10.1007/s00702-005-0443-9.

[14] G. Pfurtscheller and C. Neuper, "Future prospects of erd/ers in the context of brain-computer interface (bci) developments", Progress in brain research, vol. 159, pp. 433-437, 2006.

[15] S. Marcel and J. d. R. Millán, "Person authentication using brainwaves (eeg) and maximum a posteriori model adaptation", IEEE transactions on pattern analysis and machine intelligence, vol. 29, no. 4, pp. 743-752, 2007.

[16] S. Sanei and J. Chambers, "Fundamentals of eeg signal processing", in EEG Signal Processing. John Wiley Sons, Ltd, 2007, ch. 2, pp. 35-125, ISBN: 9780470511923. DOI: https: / / doi.org/10.1002/9780470511923.ch2. eprint: https : / / onlinelibrary. wiley. com / doi / pdf / 10 . 1002 / 9780470511923 . ch2. [Online]. Available: https : / / onlinelibrary . wiley . com / doi / abs / 10 . 1002 / 9780470511923.ch2.

[17] X. ( Zhang, W. Diao, and Z. Cheng, "Wavelet transform and singular value decomposition of eeg signal for pattern recognition of complicated hand activities", Jul. 2007, pp. 294-303, ISBN: 978-3-540-73318-8. DOI: 10.1007/978-3540-73321-8_35.

[18] M. Hannula, K. Huttunen, J. Koskelo, T. Laitinen, and T. Leino, "Comparison between artificial neural network and multilinear regression models in an evaluation of cognitive workload in a flight simulator", Computers in Biology and Medicine, vol. 38, no. 11, pp. 1163-1170, 2008, ISSN: 0010-4825. DOI: https://doi.org/10.1016/j.compbiomed.2008.09.007. [Online]. Available: https://www.sciencedirect.com/science/article/pii/S0010482508001340.

[19] M. Murugesan and R. Sukanesh, "Automated detection of brain tumor in eeg signals using artificial neural networks", in 2009 International Conference on Advances in Computing, Control, and Telecommunication Technologies, 2009, pp. 284-288. DOI: 10.1109/ACT.2009.77.

[20] P. " Antonenko, F. Paas, R. Grabner, and T. Gog, "Using electroencephalography to measure cognitive load", Educational Psychology Review, vol. 22, pp. 425-438, Dec. 2010. DOI: 10.1007/s10648-010-9130-y. 
[21] R. Brünken, T. Seufert, and F. Paas, "Measuring cognitive load", 2010.

[22] L. Chun-Lin, "A tutorial of the wavelet transform", NTUEE, Taiwan, 2010.

[23] S. A. Hosseini and M. A. Khalilzadeh, "Emotional stress recognition system using eeg and psychophysiological signals: Using new labelling process of eeg signals in emotional stress state", in 2010 international conference on biomedical engineering and computer science, IEEE, 2010, pp. 1-6.

[24] S. D. Puthankattil, P. Joseph, U. R. Acharya, and C. Lim, "Eeg signal analysis: A survey", Journal of medical systems, vol. 34, pp. 195-212, Apr. 2010. DOI: 10.1007/s10916-008-9231-z.

[25] P. Ranjan Pal and R. Panda, "Classification of eeg signals for epileptic seizure evaluation", in 2010 IEEE Students Technology Symposium (TechSym), 2010, pp. 72-76. DOI: 10.1109/TECHSYM.2010.5469195.

[26] S. Kalyuga, "Cognitive load theory: How many types of load does it really need?", Educational Psychology Review, vol. 23, no. 1, pp. 1-19, 2011.

[27] R. Panda, P. Khobragade, P. Jambhule, S. Jengthe, P. Pal, and T. Gandhi, "Classification of eeg signal using wavelet transform and support vector machine for epileptic seizure diction", Jan. 2011, pp. 405-408. DOI: 10.1109 / ICSMB.2010.5735413.

[28] M. Stecklow, M. Cagy, and A. Infantosi, "Event related synchronization to assess changes in alpha peak frequency along time during kinesthetic motor imagery", Mar. 2011. DOI: 10.1109/PAHCE.2011.5871909.

[29] M. P. G. Bhosale and S. Patil, "Classification of emg signals using wavelet transform and hybrid classifier for parkinson's disease detection", Int. J. Eng. Res. Technol, vol. 2, pp. 106-112, 2012.

[30] S. Janjarasjitt, "Characteristics of local min-max amplitude of wavelet subbands of scalp epileptic eeg", Oct. 2013, pp. 1-5, ISBN: 978-1-4799-1466-1. DOI: 10.1109/BMEiCon.2013.6687726.

[31] J. Machado, A. Balbinot, and A. Schuck, "A study of the naive bayes classifier for analyzing imaginary movement eeg signals using the periodogram as spectral estimator", in 2013 ISSNIP Biosignals and Biorobotics Conference: Biosignals and Robotics for Better and Safer Living (BRC), IEEE, 2013, pp. 14.

[32] A. Shahid, N. Kamel, A. Malik, and M. Jatoi, "Singular value decomposition (svd) as a detector of epileptic seizures in eeg signals", May 2013. DOI: 10. 1109/ICCME.2013.6548330.

[33] R. Stevens, T. Galloway, and C. Berka, "Integrating eeg models of cognitive load with machine learning models of scientific problem solving", May 2013.

[34] H. Yaacob, W. Abdul, and N. Kamaruddin, "Classification of eeg signals using mlp based on categorical and dimensional perceptions of emotions", in 2013 5th International Conference on Information and Communication Technology for the Muslim World (ICT4M), IEEE, 2013, pp. 1-6.

[35] W. Chen, Y. Wang, G. Cao, G. Chen, and Q. Gu, "A random forest model based classification scheme for neonatal amplitude-integrated eeg", Biomedical engineering online, vol. 13, no. 2, pp. 1-13, 2014. 
[36] A. S. Al-Fahoum and A. A. Al-Fraihat, "Methods of eeg signal features extraction using linear analysis in frequency and time-frequency domains", International Scholarly Research Notices, vol. 2014, 2014.

[37] R. Mahajan, C. A. Majmudar, S. Khatun, B. I. Morshed, and G. M. Bidelman, "Neuromonitor ambulatory eeg device: Comparative analysis and its application for cognitive load assessment", in 2014 IEEE Healthcare Innovation Conference (HIC), 2014, pp. 133-136. DOI: 10.1109/HIC.2014.7038892.

[38] D. C. Moos and D. Pitton, "Student teacher challenges: Using the cognitive load theory as an explanatory lens", Teaching Education, vol. 25, no. 2, pp. 127-141, 2014. DOI: 10.1080/10476210.2012.754869. eprint: https://doi. org/10.1080/10476210.2012.754869. [Online]. Available: https://doi.org/10. $1080 / 10476210.2012 .754869$.

[39] S.-H. Oh, Y.-R. Lee, and H.-N. Kim, "A novel eeg feature extraction method using hjorth parameter", International Journal of Electronics and Electrical Engineering, vol. 2, no. 2, pp. 106-110, 2014.

[40] T. Prasad and R. Tvk, Survey on eeg signal processing methods, Jan. 2014.

[41] Z. Huang, W. Xu, and K. Yu, "Bidirectional lstm-crf models for sequence tagging", arXiv preprint arXiv:1508.01991, 2015.

[42] Y. Zhang, W. Zhou, S. Yuan, and Q. Yuan, "Seizure detection method based on fractal dimension and gradient boosting", Epilepsy $\&$ Behavior, vol. 43, pp. 30-38, 2015.

[43] E. Awada, "Analysis of sfdr using power spectrum based on wavelet extraction", vol. 11, pp. 1256-1260, Mar. 2016.

[44] P. Chauhan and M. Preetam, "Brain waves and sleep science", Intl J Engg Sci Adv Research, vol. 2, no. 1, pp. 33-36, 2016.

[45] K. Kalimeri and C. Saitis, "Exploring multimodal biosignal features for stress detection during indoor mobility", in Proceedings of the 18th ACM international conference on multimodal interaction, 2016, pp. 53-60.

[46] N. Kumar and J. Kumar, "Measurement of cognitive load in hci systems using eeg power spectrum: An experimental study", Procedia Computer Science, vol. 84, pp. 70-78, 2016, Proceeding of the Seventh International Conference on Intelligent Human Computer Interaction (IHCI 2015), ISSN: 1877-0509. DOI: https: / / doi .org / 10.1016/j . procs . 2016.04.068. [Online]. Available: https://www.sciencedirect.com/science/article/pii/S1877050916300825.

[47] C. Saitis and K. Kalimeri, "Identifying urban mobility challenges for the visually impaired with mobile monitoring of multimodal biosignals", vol. 9739, Jul. 2016, pp. 616-627, ISBN: 978-3-319-40237-6. DOI: 10.1007/978-3-31940238-3_59.

[48] —, "Identifying urban mobility challenges for the visually impaired with mobile monitoring of multimodal biosignals", in International Conference on Universal Access in Human-Computer Interaction, Springer, 2016, pp. 616627.

[49] C. Amo, L. De Santiago, R. Barea, A. López-Dorado, and L. Boquete, "Analysis of gamma-band activity from human eeg using empirical mode decomposition", Sensors, vol. 17, no. 5, p. 989, 2017. 
[50] N. Bastos, D. Adamatti, and C. Billa, "Decision tree to analyses eeg signal: A case study using spatial activities", Computational Neuroscience-Communications in Computer and Information Science, vol. 720, 2017.

[51] D. Chen, S. Wan, J. Xiang, and F. S. Bao, "A high-performance seizure detection algorithm based on discrete wavelet transform (dwt) and eeg", PloS one, vol. 12, no. 3, e0173138, 2017.

[52] A. Dan and M. Reiner, "Real time eeg based measurements of cognitive load indicates mental states during learning", JEDM-Journal of Educational Data Mining, vol. 9, no. 2, pp. 31-44, 2017.

[53] M. Klepsch, F. Schmitz, and T. Seufert, "Development and validation of two instruments measuring intrinsic, extraneous, and germane cognitive load", Frontiers in Psychology, vol. 8, p. 1997, 2017, ISSN: 1664-1078. DOI: 10.3389/ fpsyg.2017.01997. [Online]. Available: https://www.frontiersin.org/article/10. 3389/fpsyg.2017.01997.

[54] R. Mehta, "Population specific template construction and brain structure segmentation using deep learning methods", PhD thesis, PhD thesis, International Institute of Information Technology Hyderabad, 2017.

[55] C. Mills, I. Fridman, W. Soussou, D. Waghray, A. M. Olney, and S. K. D’Mello, "Put your thinking cap on: Detecting cognitive load using eeg during learning", in Proceedings of the seventh international learning analytics 85 knowledge conference, 2017, pp. 80-89.

[56] S. Roussel, D. Joulia, A. Tricot, and J. Sweller, "Learning subject content through a foreign language should not ignore human cognitive architecture: A cognitive load theory approach", Learning and Instruction, vol. 52, pp. 69-79, 2017.

[57] YongchangWang and L. Zhu, "Research and implementation of svd in machine learning", in 2017 IEEE/ACIS 16th International Conference on Computer and Information Science (ICIS), 2017, pp. 471-475. DOI: 10.1109/ICIS.2017. 7960038 .

[58] A. Bablani, D. R. Edla, and S. Dodia, "Classification of eeg data using knearest neighbor approach for concealed information test", Procedia computer science, vol. 143, pp. 242-249, 2018.

[59] I. A. Jagadal and K. A. Manik, "Changes of eeg pattern in blind children-a comparative study", International Journal of Physiology, vol. 6, no. 3, pp. 7-9, 2018.

[60] Z. Koudelková and M. Strmiska, "Introduction to the identification of brain waves based on their frequency", in MATEC Web of Conferences, EDP Sciences, 2018.

[61] A. Kowalska-Koczwara and K. Stypula, "Influence of crest factor on evaluation of human perception of traffic vibration", Journal of Measurements in Engineering, vol. 6, pp. 250-255, Dec. 2018. DOI: 10.21595/jme.2018.20421.

[62] V. Saccá, M. Campolo, D. Mirarchi, A. Gambardella, P. Veltri, and F. C. Morabito, "On the classification of eeg signal by using an svm based algorithm", in Multidisciplinary approaches to neural computing, Springer, 2018, pp. 271-278. 
[63] C. Saitis, M. Z. Parvez, and K. Kalimeri, "Cognitive load assessment from eeg and peripheral biosignals for the design of visually impaired mobility aids", Wireless Communications and Mobile Computing, vol. 2018, 2018.

[64] S. Afroz and Z. H. Shimanto, "Approval the thesis / project titled " exploring cognitive load and emotional states for the visually impaired " submitted by 1", 2019.

[65] S. Afroz, Z. H. Shimanto, et al., "Exploring cognitive load and emotional states for the visually impaired", PhD thesis, Brac University, 2019.

[66] S. Aggarwal and N. Chugh, "Signal processing techniques for motor imagery brain computer interface: A review", Array, vol. 1-2, p. 100003,2019 , ISSN: 2590-0056. DOI: https://doi.org/10.1016/j.array.2019.100003. [Online]. Available: https://www.sciencedirect.com/science/article/pii/S2590005619300037.

[67] Y. Aoh, H.-J. Hsiao, M.-K. Lu, A. Macerollo, H.-C. Huang, M. Hamada, C.-H. Tsai, and J.-C. Chen, "Event-related desynchronization/synchronization in spinocerebellar ataxia type 3", Frontiers in Neurology, vol. 10, p. 822, 2019, ISSN: 1664-2295. DOI: 10.3389/fneur.2019.00822. [Online]. Available: https: //www.frontiersin.org/article/10.3389/fneur.2019.00822.

[68] Cabañero, Hervás, González, Fontecha, Mondéjar, and J. Bravo, "Analysis of cognitive load using eeg when interacting 40 errors18 warnings with mobile devices", Proceedings, vol. 31, p. 70, Nov. 2019. DOI: 10.3390/proceedings2019031070.

[69] N. Friedman, T. Fekete, K. Gal, and O. Shriki, "Eeg-based prediction of cognitive load in intelligence tests", Frontiers in human neuroscience, vol. 13, p. 191, 2019.

[70] R. Kabir, M. Rashid, and M. Or, "Cognitive load detection of vision impaired in the inward places using bio-signal", PhD thesis, Brac University, 2019.

[71] S. Sepp, S. J. Howard, S. Tindall-Ford, S. Agostinho, and F. Paas, "Cognitive load theory and human movement: Towards an integrated model of working memory", Educational Psychology Review, pp. 1-25, 2019.

[72] T. Yin and X. Chen, "Research on broadband dither technique in signal acquisition", The Journal of Engineering, vol. 2019, Mar. 2019. DOI: 10.1049/ joe.2018.9155.

[73] R. Atangana, D. Tchiotsop, G. Kenne, and L. Chanel, "Eeg signal classification using lda and mlp classifier", Health Informat. Int. J., vol. 9, no. 1, pp. 14-32, 2020 .

[74] V. Bajaj, S. Taran, S. K. Khare, and A. Sengur, "Feature extraction method for classification of alertness and drowsiness states eeg signals", Applied Acoustics, vol. 163, p. $107224,2020$.

[75] P. Boonyakitanont, A. Lek-Uthai, K. Chomtho, and J. Songsiri, "A review of feature extraction and performance evaluation in epileptic seizure detection using eeg", Biomedical Signal Processing and Control, vol. 57, p. 101 702, 2020.

[76] L. Cheng, D. Li, G. Yu, Z. Zhang, X. Li, and S. Yu, "A motor imagery eeg feature extraction method based on energy principal component analysis and deep belief networks", IEEE Access, vol. PP, pp. 1-1, Jan. 2020. DOI: 10.1109/ ACCESS.2020.2969054. 
[77] M. Cukić, M. Stokić, D. Pokrajac, and S. Slobodan, "The successful discrimination of depression from eeg could be attributed to proper feature extraction and not to a particular classification method", Cognitive Neurodynamics, Feb. 2020.

[78] V. Gupta and R. B. Pachori, "Classification of focal eeg signals using fbse based flexible time-frequency coverage wavelet transform", Biomedical Signal Processing and Control, vol. 62, p. 102 124, 2020.

[79] J. Janssen and P. A. Kirschner, "Applying collaborative cognitive load theory to computer-supported collaborative learning: Towards a research agenda", Educational Technology Research and Development, vol. 68, no. 2, pp. 783805, Apr. 2020, ISSN: 1556-6501. DOI: 10.1007/s11423-019-09729-5. [Online]. Available: https://doi.org/10.1007/s11423-019-09729-5.

[80] Z. Li, L. Zhang, F. Zhang, R. Gu, W. Peng, and L. Hu, "Demystifying signal processing techniques to extract resting-state eeg features for psychologists", Brain Science Advances, vol. 6, no. 3, pp. 189-209, 2020.

[81] Z. Luo, X. Lu, and X. Xi, "Eeg feature extraction based on a bilevel network: Minimum spanning tree and regional network", Electronics, vol. 9, no. 2, p. 203, 2020.

[82] —, "Eeg feature extraction based on a bilevel network: Minimum spanning tree and regional network", Electronics, vol. 9, no. 2, p. 203, 2020.

[83] R.-G. Lupu, O. Mitrut, A. Stan, F. Ungureanu, K. Kalimeri, and A. Moldoveanu, "Cognitive and affective assessment of navigation and mobility tasks for the visually impaired via electroencephalography and behavioral signals", Sensors, vol. 20, no. 20, p. 5821, 2020.

[84] R. Nawaz, K. H. Cheah, H. Nisar, and V. V. Yap, "Comparison of different feature extraction methods for eeg-based emotion recognition", Biocybernetics and Biomedical Engineering, vol. 40, no. 3, pp. 910-926, 2020.

[85] R. Ramos-Aguilar, J. A. Olvera-López, I. Olmos-Pineda, and S. SánchezUrrieta, "Feature extraction from eeg spectrograms for epileptic seizure detection", Pattern Recognition Letters, vol. 133, pp. 202-209, 2020.

[86] Q. Xiong, X. Zhang, W.-F. Wang, and Y. Gu, "A parallel algorithm framework for feature extraction of eeg signals on mpi", Computational and Mathematical Methods in Medicine, vol. 2020, 2020.

[87] A. Nandi, F. Xhafa, L. Subirats, and S. Fort, "Real-time emotion classification using eeg data stream in e-learning contexts", Sensors, vol. 21, no. 5, p. 1589, 2021.

[88] D. Parbat and M. Chakraborty, "A novel methodology to study the cognitive load induced eeg complexity changes: Chaos, fractal and entropy based approach", Biomedical Signal Processing and Control, vol. 64, p. 102 277, 2021.

[89] T. Tamanna and M. Z. Parvez, "Cognitive load measurement based on eeg signals", in The Science of Emotional Intelligence, IntechOpen, 2021. 